\title{
Low Cerebral Exposure Cannot Hinder the Neuroprotective Effects of Panax Notoginsenosides ${ }^{[\mathbb{S}}$
}

\author{
Haofeng Li, ${ }^{1}$ Jingcheng Xiao, ${ }^{1}$ Xinuo Li, Huimin Chen, Dian Kang, Yuhao Shao, Boyu Shen, \\ Zhangpei Zhu, Xiaoxi Yin, Lin Xie, Guangji Wang, and Yan Liang
}

Key Laboratory of Drug Metabolism and Pharmacokinetics, State Key Laboratory of Natural Medicines, China Pharmaceutical University, Nanjing, China

Received September 1, 2017; accepted October 13, 2017

\begin{abstract}
A bidirectional route of communication between the gastrointestinal tract and the central nervous system, termed the "gut-brain axis," is becoming increasingly relevant to treatment of cerebral damage. Panax Notoginsenoside extract (PNE) is popular for prevention and treatment of cardio-cerebrovascular ischemic diseases although plasma and cerebral exposure levels are extremely low. To date, the mechanisms underlying the neuroprotective effects of PNE remain largely unknown. In the present study, the neuroprotective effects of PNE were systematically studied via investigation of the regulation by PNE of the gastrointestinal microbial community and $\gamma$ aminobutyric acid (GABA) receptors. The results demonstrated that pretreatment with PNE exerted a remarkable neuroprotective effect on
\end{abstract}

focal cerebral ischemia/reperfusion (I/R) injury in rats, and the efficiency was attenuated in germ-free rats. Pretreatment with PNE could significantly prevent downregulation of Bifidobacterium longum (B.L) caused by I/R surgery, and colonization by B.L could also exert neuroprotective effects. More importantly, both PNE and B.L could upregulate the expression of GABA receptors in the hippocampus of $\mathrm{I} / \mathrm{R}$ rats, and coadministration of a GABA-B receptor antagonist could significantly attenuate the neuroprotective effects of PNE and B.L. The study above suggests that the neuroprotective effects of PNE may be largely attributable to its regulation of intestinal flora, and oral treatment with B.L was also useful in therapy of ischemia/reperfusion injury (I/R) by upregulating GABA-B receptors.

\section{Introduction}

Worldwide, ischemic brain injury is a major cause of dementia, disability, and death, and most of the survivors suffer from long-term sequelae, including seizures, mental retardation, and neurologic deficits (Donnan et al., 2008). Reperfusion of ischemic areas can exacerbate brain damage through release of free radicals and cytokines, platelet activation, and apoptosis (Doyle et al., 2008). In the past decade, the influence of the gastrointestinal (GI) tract on the occurrence, development, and treatment of brain diseases had been noted in humans, and a bidirectional communication route between the GI tract and the central nervous system (CNS) was termed the "gut-brain axis" (Maqsood and Stone, 2016). The main bidirectional communication of the gut-brain axis mainly occurs via the vagus nerve, gut hormones [e.g., $\gamma$-aminobutyric acid (GABA), neuropeptide Y, dopamine], and gut microbiota molecules (e.g., short-chain fatty acids, tryptophan) (Braniste et al., 2014; Clark and Mach, 2016; Eisenstein, 2016). Recent interest in gut microbiota has revealed that in addition to reflecting the body state and energy

This study was supported by the National Nature Science Foundation of China [Grants 81374054, 81573559, 81530098], the Nature Science Foundation of Jiangsu Province [Grant BK20171395], and the Outstanding Youth fund of State Key Laboratory of Natural Medicines [Grant SKLNMZZJQ201602].

${ }^{1}$ H.L. and J.X. contributed equally to this work.

https://doi.org/10.1124/dmd.117.078436.

S This article has supplemental material available at dmd.aspetjournals.org. metabolism, colonic bacteria can influence curative efficacy of drugs (Vyas and Ranganathan, 2012). To date, more than 100 trillion microorganisms have been found in the human gut (Yang et al., 2009). Gut microbiota affect numerous biologic functions and are linked to pathogenesis of various diseases such as obesity, cancer, and liver cirrhosis (Lee and Hase, 2014). The interactions among the intestinal microbiota, GI tract, and CNS constitute the microbiome-gut-brain axis, and research on how the microbiome-gut-brain axis influences neurologic conditions may provide insight into disease etiology and treatment strategy (Sampson and Mazmanian, 2015; Foster et al., 2016; Sampson et al., 2016). For example, consumption of probiotics can dramatically affect sustained attention performance as well as brain activity during social cognition (Tillisch et al., 2013). Recent preclinical and clinical studies have identified Bifidobacterium longum (B.L) and Lactobacillus helveticus (L.H) as putative psychobiotics with great impact on stressrelated brain-gut-axis disorders, behaviors, physiology, and cognitive performance (Ohsawa et al., 2015).

In the past few years, researchers have focused on neural, immune, endocrine, and metabolic pathways as probable mediators in this bidirectional communication (El Aidy et al., 2015; Mayer et al., 2015). GABA, which is a well known neurotransmitter in the development and modulation of neuropathy, can be produced in large quantities by several types of bacteria in the GI tract (Zheng et al., 2007). It has been reported that Lactobacillus brevis (L.B) FPA 3709 (prodigious producer of GABA) employs GABA-enriched soybean milk and can

ABBREVIATIONS: ANOVA, analysis of variance; B.L, Bifidobacterium longum; BDNF, brain-derived neurotrophic factor; CCA, common carotid artery; CFU, colony-forming units; CNS, central nervous system; GABA, $\gamma$-aminobutyric acid; Gl, gastrointestinal; I/R, ischemia/reperfusion; IL-1 $\beta$, interleukin-1 $\beta$; IL-6, interleukin-6; L.B, Lactobacillus brevis; L.H, Lactobacillus helveticus; L.R, Lactobacillus rhamnosus; PCR, polymerase chain reaction; PGF, pseudo-germ-free; PNE, Panax Notoginsenoside extract; $R_{1}$, notoginsenoside $R_{1} ; R_{1}$, ginsenoside $R b_{1}$; $R d$, ginsenoside $R d$; $R e$, ginsenoside $\mathrm{Re} ; \mathrm{Rg}_{1}$, ginsenoside $\mathrm{Rg}_{1}$; TNF- $\alpha$, tumor necrosis factor- $\alpha$; TTC, 2,3,5-triphenyltetrazolium chloride. 
significantly reduce rat depressive-like behavior as effectively as the classic antidepressant fluoxetine (Ko et al., 2013). In another study, the ability of orally administered Lactobacillus rhamnosus (L.R) to reduce anxiety- and depressive-like behaviors in mice was shown to be mediated by GABA receptor expression in the brain, with changes in levels of GABA-A $\alpha 2$ mRNA in brain regions associated with the specific behavior (Bravo et al., 2011). Effects of GABA are typically mediated through two major classes of receptors, GABA-A and GABA-B (Helm et al., 2005). Interestingly, regulation of GABA-A receptor subunits can significantly affect fear-related behaviors (Bravo et al., 2011), and activation of metabotropic GABA-B receptors can trigger secretion of endogenous brain-derived neurotrophic factor (BDNF) (Fiorentino et al., 2009), which exerts neuroprotective effects against glutamate toxicity in hippocampal neurons.

Herbal traditional Chinese medicines (TCMs) are known to exert wide pharmaceutical effects in a cooperative way with multiple components and targets, yet its efficiency is poorly documented and always described in general terms without detailed characterization of herbs and diseases (Ma et al., 2014). The overall growing popularity of herbal TCMs has led to substantial interest in laboratory and clinical studies to evaluate their efficiency and pharmacological mechanisms (Teschke et al., 2015). Panax Notoginsenoside (PNE), which is an extract from the traditional Chinese herb Panax notoginseng, has been commonly used to treat cardiocerebrovascular diseases for thousands of years (Huang et al., 2015). In addition, PNE has a curative effect in treatment of common aural vertigo by promoting blood circulation to dissipate blood stasis. The main components of PNE, including notoginsenoside R1 (R1), ginsenoside $R b_{1}\left(R b_{1}\right)$, ginsenoside $R d(R d)$, ginsenoside $R e(R e)$, and ginsenoside $\operatorname{Rg} 1$ ( $\operatorname{Rg} 1)$, have been proven to be bioactive for prevention and treatment of cardiovascular and cerebrovascular diseases (Meng et al., 2012). However, previous studies on the pharmacokinetics of Panax Notoginsenosides showed that bioavailability of the main notoginsenosides $\left(\mathrm{R}_{1}, \mathrm{Rd}, \mathrm{Re}, \mathrm{Rg} 1, \mathrm{Rb} 1, \mathrm{Rb} 2\right)$ was extremely poor $(<2 \%)$, and notoginsenoside exposure in the systemic circulation was quite low (Xu et al., 2014; Kim et al., 2015). For instance, notoginsenosides do not readily cross the blood-brain barrier owing to poor membrane permeability, and concentrations of notoginsenosides in the rat cortex, striatum, hypothalamus, medulla oblongata, and hippocampus were much lower than $100 \mathrm{ng} / \mathrm{g}$ (Guo et al., 2014). Thus, the pharmacodynamic and pharmacokinetic profiles of notoginsenosides are contradictory, and their curative effect on cerebral ischemic injury remains largely unexplored.

In the present study, we aimed to investigate the neuroprotective effects and mechanism of PNE on ischemia-reperfusion (I/R)-induced focal cerebral ischemia via investigation of PNE's regulation of I/Rrelated brain-gut-axis disorders. The results showed that pretreatment with PNE could significantly upregulate the relative abundance of B.L in $\mathrm{I} / \mathrm{R}$ rats, and colonization of B.L could also play a protective role in focal cerebral ischemia. Finally, both PNE and B.L could upregulate expression of GABA receptors, and their neuroprotective effects could be greatly attenuated by a GABA-B receptor antagonist.

\section{Materials and Methods}

\section{Chemicals and Standards}

Panax Notoginsenoside extraction and authentic standards of notoginsenoside $\mathrm{R} 1, \mathrm{Rd}, \mathrm{Rb} 2, \mathrm{Rg} 1, \mathrm{Rb} 1$, Re were purchased from Department of Nature Medical Chemistry in Jilin University (Changchun, Jilin, China). Digoxin [internal standard (IS)] was purchased from Jiangsu Institute for Food and Drug Control (Nanjing, Jiangsu, China). Saclofen, streptomycin, neomycin sulfate, and 2,3,5-triphenyltetrazolium chloride (TTC) were purchased from Sigma-Aldrich
Corporation (Sigma Aldrich, St. Louis, MO). Bicuculine was purchased from Aladdin Chemical Reagent Co., Ltd (Shanghai, China). Bacteria Genomic DNA Extraction kit was purchased from Takara Bio Inc. (Kusatsu, Shiga, Japan). Both anti-GABA B receptor 1 (ab166604) and anti-GABA B receptor 2 (ab52248) were purchased from Abcam (Cambridge, UK). SYBR Green Supermix RT-PCR Kit was purchased from Bio-Rad (Hercules, CA). Deionized water was purified using a Milli-Q Ultrapure water system with the water outlet operating at $18.2 \mathrm{M} \Omega$ (MilliporeSigma, Billerica, MA). Other chemicals and solvents were all of analytical grade.

\section{Animals and Treatments}

Animals. Healthy Sprague-Dawley rats $(220 \pm 10 \mathrm{~g})$ were purchased from Shanghai Super-B+K Laboratory Animal Corp. Ltd. (B+K, Shanghai, China) and acclimated for 5 days before use. All animals were kept in an environmentally controlled breeding room under controlled temperature $\left(20-24^{\circ} \mathrm{C}\right)$ and relative humidity $(40 \%-70 \%)$ with a 12 -hour light/dark cycle. Standard diet and water were provided to the rats ad libitum. All procedures in this study were conducted in accordance with the Guide for the Care and Use of Laboratory Animals as adopted and promulgated by the Committee on the Ethics of Animal Experiments of China Pharmaceutical University (Nanjing, Jiangsu, China). Ethical procedures were conducted under Reduction, Replacement, and Refinement (the three Rs rule).

Preparation of I/R Model Rats. Rats were first anesthetized using $2 \mathrm{ml}$ of enflurane in an ether jar and maintained with $10 \%$ chloral hydrate $\left(400 \mathrm{mg} \mathrm{kg}^{-1}\right.$, i.p.). Anesthetized rats underwent surgery for permanent occlusion of the middle cerebral artery as reported previously (Gerriets et al., 2003). In brief, the right proximal common carotid artery (CCA), the external carotid artery (ECA), and the occipital artery (OA) were ligated by silk sutures, and the internal carotid artery (ICA) was occluded by a microvascular clip. Then a 4-0 nylon monofilament suture with a thin silicon coat was inserted into the CCA. After removal of the microvascular clip, the monofilament suture was gently slipped into the ICA $18 \mathrm{~mm}$ beyond the bifurcation of the CCA, which resulted in permanent occlusion of the middle cerebral artery occlusion (MCAO). Reperfusion was induced at 2 hours after MCAO via withdrawal of the monofilament. Sham-operated animals were subjected to the same surgical procedures except that the CCA, ECA, and OA were not ligated.

Preparation and Evaluation of Pseudo-Germ-Free Rat Model. For the preparation of pseudo-germ-free (PGF) rats, an antibiotic cocktail was prepared by dissolving neomycin sulfate and streptomycin in water, and Sprague-Dawley rats were orally administered the antibiotic cocktail $(100 \mathrm{mg} / \mathrm{kg}$ per each antibiotic) twice a day continuously for 6 days. The experiments were performed 2 days later after the final administration.

Drug Administration. PNE was dissolved in normal saline and intragastrically administrated to rats at a dose of $100 \mathrm{mg} / \mathrm{kg}$ for 7 consecutive days before I/R surgery. The rats in the vehicle group were administrated saline in the same way.

Neurologic Evaluation for the I/R Model Rats. Neurologic evaluation was performed at 26 hours after induction of ischemia and scored on a 5-point scale according to previous reports (Longa et al., 1989): A score of 0 meant no neurologic deficit, a score of 1 (failure to extend left forepaw fully) indicated a mild focal neurologic deficit, a score of 2 (circling to the left) was a moderate focal neurologic deficit, and a score of 3 (falling to the left) demonstrated a severe focal deficit; rats with a score of 4 could not walk spontaneously and had a depressed level of consciousness.

TTC Assessment of Infarct Size. Cerebral infarct size was assessed with TTC staining method (Joshi et al., 2004). After 26 hours of I/R (2-hour ischemia and 24-hour reperfusion), the rat brains were quickly isolated and sectioned into consecutive 2-mm-thick coronal slices with a cryomicrotome (Leica CM1950; Nussloch, Germany). The brain slices were immediately immersed in $0.1 \%$ TTC medium (TTC, $0.125 \%$ w:v in $62.5 \mathrm{mM}$ Tris- $\mathrm{HCl}, 13 \mathrm{mM} \mathrm{MgCl} 2,1.5 \%$ dimethylformamide) at $37^{\circ} \mathrm{C}$ for 15 minutes and incubated for another 15 minutes after overturning the slices. The stained slices were then washed in phosphatebuffered saline $(0.01 \mathrm{M})$ for 5 minutes and immobilized in a buffered formaldehyde solution for 24 hours. After immobilization, color images were captured using a video camera. Further analysis was conducted to calculate the infarct volume using the Image-J analysis software according to the following equation: $\left[\left(\mathrm{V}_{\mathrm{C}}-\mathrm{V}_{\mathrm{L}}\right) / \mathrm{V}_{\mathrm{C}}\right]^{*} 100$. In the formula, $\mathrm{V}_{\mathrm{C}}$ is the total volume of the sphere, and $\mathrm{V}_{\mathrm{L}}$ is the volume of noninfarcted sphere. 
Determination of Proinflammatory Cytokines, BDNF, and GABA in Rat Hippocampus. The tumor necrosis factor- $\alpha$ (TNF- $\alpha$ ), interleukin-6 (IL-6), and interleukin-1 $\beta$ (IL-1 $\beta$ ) levels in brain tissues were quantified using the ExCell ELISA kits (ExCell Biology, Shanghai, China) following the manufacturer's instructions. The BDNF and GABA were measured using the USCN ELISA kits (Wuhan USCN Business Co., Ltd, Wuhan, Hubei, China) according to the manufacturer's instructions.

\section{Gut Microbe Gene Sequencing}

The procedures of gut microbe gene sequencing were same as our previous report (Gerriets et al., 2003), and three steps were involved in this process.

DNA Extraction and Polymerase Chain Reaction Amplification. Total genome DNA of intestinal microbiota was extracted using hexadecyl trimethyl ammonium bromide, and the DNA concentration was diluted to $1 \mathrm{ng} \mu \mathrm{l}^{-1}$ using sterile water. The distinct regions (16SV4/16SV3/16SV3-V4/16SV4-V5) of $16 \mathrm{~S}$ rRNA genes were amplified using specific primer (341F: CCTAYGGGRBGCASCAG, 806R: GGACTACNNGGGTATCTAAT). The reaction system (30 $\mu \mathrm{l})$ consisted of each primer $(10 \mu \mathrm{M}, 0.5 \mu \mathrm{l})$, Es Taq $(15 \mu \mathrm{l})$, and DNA template $(1 \mu \mathrm{l})$. The polymerase chain reaction (PCR) reactions were performed with Phusion High-Fidelity PCR Master Mix (New England BioLabs, Ipswich, MA), and thermal cycling consisted of initial denaturation at $98^{\circ} \mathrm{C}$ for 1 minute, followed by 30 cycles of denaturation at $98^{\circ} \mathrm{C}$ for 10 seconds, annealing at $50^{\circ} \mathrm{C}$ for 30 seconds, and elongation at $72^{\circ} \mathrm{C}$ for 30 seconds. The PCR products were mixed with an equal volume of $2 \times$ loading buffer and electrophoresis was operated on 2\% agarose gel (contained SYB green) for detection. Samples with bright main strip between 400 and 450 bp were chosen and mixed in equidensity ratios. Then the mixture PCR products were purified with Qiagen Gel Extraction Kit (Valencia, CA).

MiSeq Sequencing of 16S rRNA Gene Amplicons. The 16S rRNA gene amplicons were performed using Illumina MiSeq sequencing at Novogene Bioinformatics Technology Co., Ltd (Novogene, Beijing, China). Sequencing libraries were generated using TruSeq DNA PCR-Free Sample Preparation Kit (Illumina, San Diego, CA) according to the manufacturer's recommendations. The library quality was evaluated on the Qubit @ 2.0 Fluorometer (Thermo Fisher Scientific, Waltham, MA) and Agilent Bioanalyzer 2100 system (Agilent Technologies, Palo Alto, CA). Finally, the library was sequenced on an IlluminaHiSeq2500 platform (Illumina).

Data Analysis. Paired-end reads were merged using FLASH v1.2.7 (http:// ccb.jhu.edu/software/FLASH/) on the basis of overlapping regions within pairedend reads. Quality filtering of the raw tags was performed to obtain the highquality clean tags according to the QIIME (V1.7.0, http://qiime.org/index.html) quality control process. Tags were compared with the reference database [Genomes OnLine Database (GOLD), US Department of Energy Joint Genome Institute (DOE JGI), Walnut Creek, CA] using UCHIME logorithm to detect chimera sequences. Sequence analysis was performed using UPARSE software (version 7.0.1001, http://drive5.com/uparse/). Sequences with $\geq 97 \%$ similarity were assigned to the same Operational Taxonomic Units (OTUs). The representative sequence for each OTU was screened for further annotation. For each representative sequence, the Greengenes Database (http://greengenes.lbl. gov/Download/) was used to annotate taxonomic information on the basis of the RDP classifier (version 2.2, https://sourceforge.net/projects/rdp-classifier/).

\section{B.L Cultivation and Colonization to Rats}

B.L (ATCC 15707) was purchased from China Center of Industrial Culture Collection and grown under anaerobic conditions in reinforced clostridium medium (RCM), which containing $0.002 \%$ polymyxin B. Bacterial cells were harvested at 48 hours and pelleted by centrifugation for 15 minutes at $5000 \mathrm{~g}$ $\left(4^{\circ} \mathrm{C}\right)$, further resuspended at a concentration of $1 \times 10^{10} \mathrm{ml}^{-1}$ in RCM, and kept in frozen aliquots until use. To observe the neuroprotective effects of B.L, rats were gavaged with $1 \times 10^{9}$ colony-forming units (CFU) of bacteria (suspended in the RCM) once per day for 15 consecutive days before I/R surgery.

\section{PCR Analysis of GABA Receptors}

Total RNA of rat Hippocampus was extracted using Exiqon kit (Takara) and reverse transcribed to cDNA using PrimeScript RT reagent kit (Takara). Expression levels of the GABA receptors $(\mathrm{A} \alpha 2, \mathrm{~A} \beta 2, \mathrm{~A} \gamma 2, \mathrm{~B} 1 \mathrm{~b}$, and $\mathrm{B} 2)$ in hippocampus were measured by real time PCR, and the primer sequences are list in Supplemental Table 1. Each experiment was performed in triplicate with at least three independent samples. Data sets were evaluated using $t$ test or one-way analysis of variance (ANOVA) followed by Tukey's post hoc test, and expressed as mean \pm S.D. Statistically significant difference was set at $P<0.05$.

\section{Western Blotting Analysis of GABA Receptors}

For Western blot analysis, rat hippocampus was isolated and homogenized in ice-cold lysis buffer containing $1 \%$ SDS, $5 \mathrm{mM}$ EDTA, $50 \mathrm{mM}$ Tris ( $\mathrm{pH} 8.3$ ), and a cocktail of protease and phosphatase inhibitors for 30 minutes and then ultrasonicated for 60 -second intervals in an ice bath. Homogenates were centrifuged at $13,000 \mathrm{~g}$ for 15 minutes at $4^{\circ} \mathrm{C}$. Protein concentrations were measured using a BCA protein assay kit (Pierce/Thermo Fisher Scientific) according to the manufacturer's instructions. The samples were diluted using Laemmli buffer (2\% SDS, $20 \%$ glycerol, $62.5 \mathrm{mM}$ Tris $\mathrm{HCl}$, $5 \%$ 2-mercaptoethanol, $0.002 \%$ bromophenol blue, $300 \mathrm{mM}$ dithiothreitol, $\mathrm{pH}$ 6.8), and then denatured by heating to $95^{\circ} \mathrm{C}$ for 5 minutes. The wells of a $10 \%$ acrylamide-bisacrylamide gel were loaded with $50 \mu \mathrm{g}$ of protein. The voltage of the electrophoresis instrument was set to $60 \mathrm{~V}$. When the protein sample entered the separation gel, the voltage was increased to $90 \mathrm{~V}$. Proteins were subsequently transferred to a membrane (orb90491; Biobyt, San Francisco, CA), blocked in 5\% nonfat milk with $0.1 \%$ Tween- 20 in Trisbuffer for 1 hour at room temperature, and incubated overnight in the primary antibodies at $4^{\circ} \mathrm{C}$.

The membrane was washed $(4 \times 10$ minutes $)$, and then incubated in appropriate secondary antibodies: anti-rabbit HRP (KGAA35, KeyGEN BioTECH, Nanjing, China) diluted $1: 10,000$ for 1 hour at $4^{\circ} \mathrm{C}$; and bound immunoglobulins were visualized with the SuperSignal West Pico chemiluminescent substrate (Pierce/Thermo Fisher Scientific) on G:BOX chemiXR5. Grayscale analysis was carried out using Gel-Pro32 software.

\section{The Influence of GABA Receptor Antagonists on PNE Efficacy}

The rats in vehicle group were treated with isometric medium. To investigate the blunting effects of GABA-A receptor and GABA-B receptor antagonists on the neuroprotective effect of PNE and B.L, bicuculline (GABA-A receptor antagonist, $0.2 \mathrm{mg} \mathrm{kg}^{-1}$ ) was administrated to rats via intraperitoneal injection, and saclofen (GABA-B receptor antagonist, $0.1 \mathrm{mg} \mathrm{kg}^{-1}$ ) was intravenously administrated to rats at 30 minutes before I/R surgery.

\section{Determination of Notoginsenoside Concentrations in Normal and I/R Rat} Brain

The method for simultaneous quantitative analysis of multiple notoginsenosides (notoginsenoside $\mathrm{R} 1, \mathrm{Rg} 3, \mathrm{Rd}, \mathrm{Rg} 2, \mathrm{Rb} 2, \mathrm{Rf}, \mathrm{Rg} 1, \mathrm{Rb} 1$, and $\mathrm{Re}$ ) was developed on the basis of UFLC-MS/MS in our previous study (Zhou et al., 2015). The method was fully validated in accordance with US FDA Bioanalytical Method Validation guidance with respect to selectivity, linearity, sensitivity, accuracy, precision, matrix effect, recovery, stability, etc. (Guidance for Industry, Bioanalytical Method Validation, 2001) The results demonstrated that the method could meet the requirement for determination of ginsenosides in biologic matrices, and the specific steps were as follows.

Sample Preparation. Both I/R model and normal rats were administrated with PNE intragastrically at a dose of $100 \mathrm{mg} \mathrm{kg}$. The rat plasma and brain specimens were quickly collected at 30 minutes and 2 and 10 hours post-dose and frozen at $-80^{\circ} \mathrm{C}$. Before analysis, brain tissues were homogenized and extracted using $n$-butanol as follows: 1 ) To each tube containing $100 \mu 1$ of homogenate, $10 \mu \mathrm{l}$ of internal standard (digoxin, $0.1 \mu \mathrm{g} \mathrm{ml}^{-1}$ ) and $1.0 \mathrm{ml}$ of $n$-butanol was added, 2) the mixture was vortex-extracted for 2 minutes and centrifuged for 5 minutes at $10,000 \mathrm{~g}$, and 3 ) the $n$-butanol extract was evaporated to dryness in a rotary evaporator and the residue was reconstituted in $200 \mu \mathrm{l}$ of acetonitrile.

Instrument, Parameters, and Conditions. The ultra-fast liquid chromatography-triple quadrupole mass spectrometer system (UFLCMS/MS-8050 system), composed of a Shimadzu 30 AD liquid chromatography system and a 8050 triple quadrupole mass spectrometer (Kyoto, Japan), was used to quantitatively analyze notoginsenosides in biologic matrices. Data acquisition was performed using the LabSolutions LCMS Ver. 5.6 software (Shimadzu). Chromatographic separation was achieved on $\mathrm{C} 18$ reversed phase 
LC column (Thermo ODS $5 \mathrm{~m} 50 \mathrm{~mm} \times 2.1 \mathrm{~mm} \mathrm{I.D.).} \mathrm{The} \mathrm{electrospray}$ ionization source was operated in negative mode. The optimized MRM parameters are list in Supplemental Table 2, and the other operating parameters were as follows: $250^{\circ} \mathrm{C}$ of desolvation temperature, $400^{\circ} \mathrm{C}$ of heat block temperature, 31 $\mathrm{min}^{-1}$ of nebulizer gas flow rate, and $51 \mathrm{~min}^{-1}$ of drying gas flow rate.

\section{Results}

Intracephalic and Plasma Concentrations of Notoginsenosides in Normal and I/R Rats. To elucidate deposition and protective mechanisms of PNE in the brain, intracephalic concentrations of six main PNE components ( $\mathrm{R} 1, \mathrm{Rd}, \mathrm{Re}, \mathrm{Rg} 1, \mathrm{Rb} 1, \mathrm{Rb} 2)$ were determined using a UFLC-MS/MS-8050 system (Shimadzu). As illustrated in Fig. 1, A to $\mathrm{C}$, detectable levels of $\mathrm{R} 1, \mathrm{Rd}, \mathrm{Re}, \mathrm{Rg} 1, \mathrm{Rb} 1$, and $\mathrm{Rb} 2$ were observed in rat brains collected at 2 hours, and intracephalic concentrations of the rats in the I/R group were higher than those in the normal group. For brain tissues collected at 30 minutes, only R1, Rg1, and Rb1 could be quantified, and the concentrations of $\mathrm{Rd}, \mathrm{Re}$, and $\mathrm{Rb} 2$ were lower than $1 \mathrm{ng} \mathrm{g}^{-1}$. At 10 hours, only trace levels of $\mathrm{R} 1$ and $\mathrm{Rb} 1$ were detectable $\left(\sim 1 \mathrm{ng} \mathrm{g}^{-1}\right)$ in the normal and I/R model rat brains. In addition, the combined molar concentration of $\mathrm{R} 1, \mathrm{Rd}, \mathrm{Re}, \mathrm{Rg} 1, \mathrm{Rb} 1$, and $\mathrm{Rb} 2$ were calculated to express the exposure of $\mathrm{PNE}$ in rat brains. At 30 minutes, the combined molar concentrations (CMCs) of notoginsenosides in normal and I/R model rat brains were 0.0867 and $0.1311 \mathrm{nmol} / \mathrm{g}$, respectively. The CMCs were 0.0867 and $0.1311 \mathrm{nmol} / \mathrm{g}$ in normal and I/R model rat brains collected at 2 hours, which were significantly higher than those collected at 30 minutes. At 10 hours, the CMCs decreased to $0.003 \mathrm{nmol} / \mathrm{g}$ in normal and $\mathrm{I} / \mathrm{R}$ rat brains.

The plasma concentrations of notoginsenosides were also measured in the present study (Fig. 1, D-F). Obviously, the concentrations of R1, $\mathrm{Rd}, \mathrm{Re}, \mathrm{Rg} 1, \mathrm{Rb} 1$, and $\mathrm{Rb}$ in rat plasma were significantly higher than those in rat brains. To demonstrate the distribution characteristics more clearly, the concentration ratios of brain to plasma were calculated for

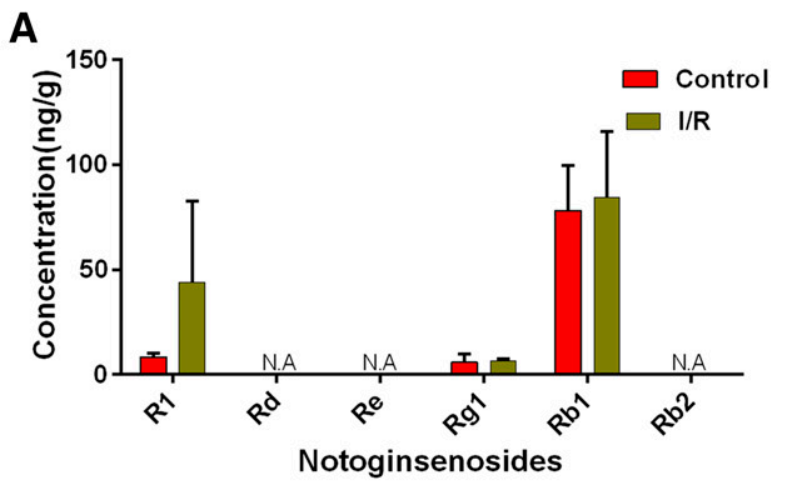

B

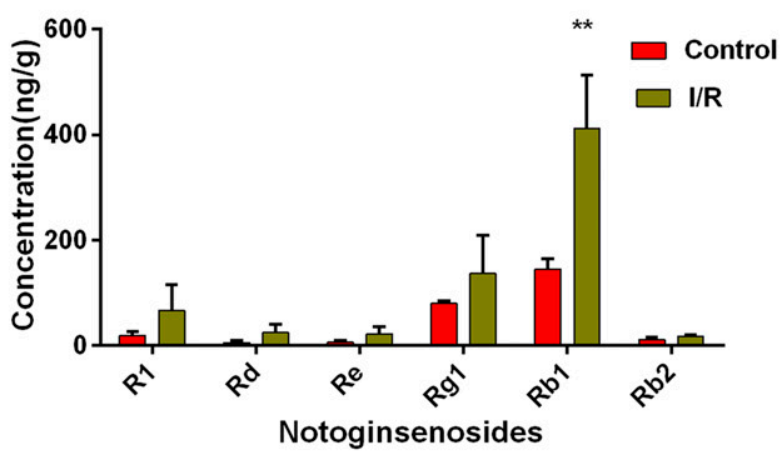

C

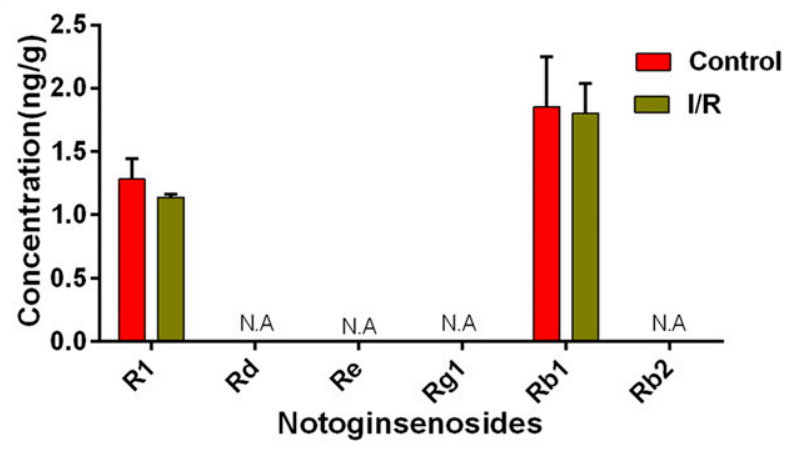

$E$

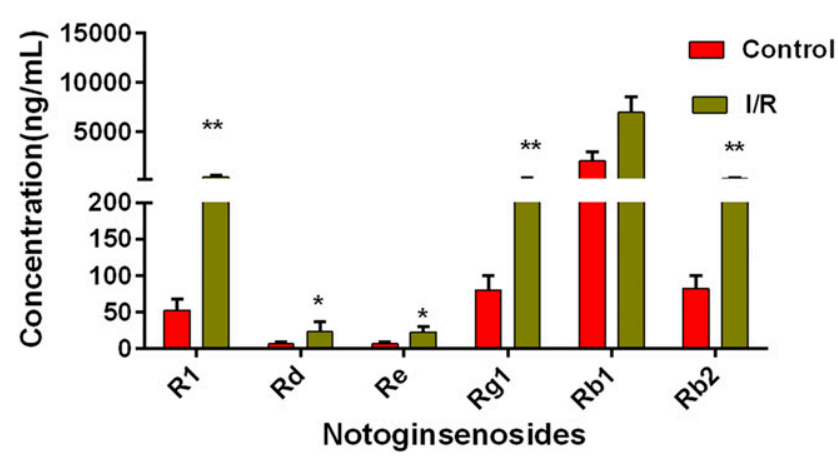

D

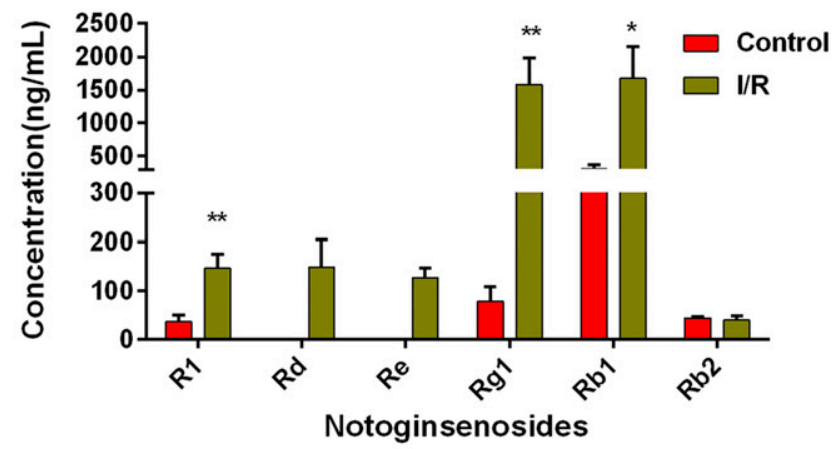

$\mathbf{F}$

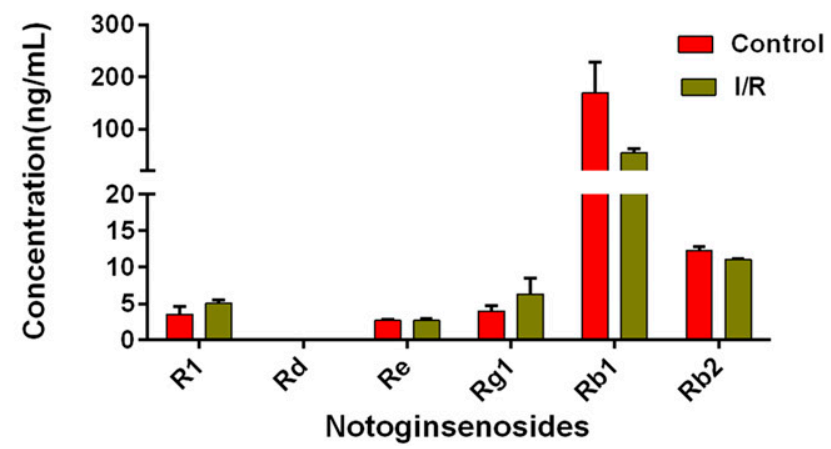

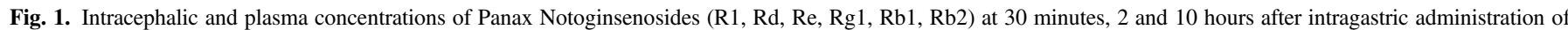

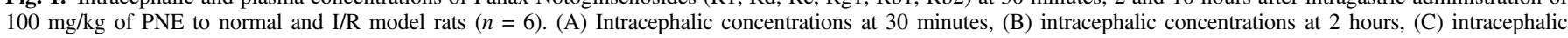

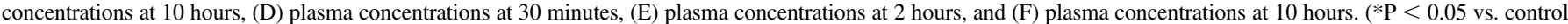
group, $* * \mathrm{P}<0.01$ vs. control group). 
the notoginsenosides. All the ratios were found to be below 1.0 (Supplemental Table 3). In sum, the exposure of notoginsenosides was quite low in the rat brain.

Neuroprotective Effects of PNE in Rats with Focal Cerebral Ischemia. To investigate neuroprotective effects of PNE, infarct volume in the brain after reperfusion for 24 hours was estimated using TTC staining. As shown in Fig. 2A, cerebral infarcts were pronounced after the I/R model was established (2-hour ischemia and 24-hour reperfusion); the cerebral infarct volume was $37.74 \% \pm 3.94 \%$ in the $\mathrm{I} / \mathrm{R}$ group (Fig. 2B). Compared with the I/R group, infarct size in the PNE treatment group was significantly decreased with a cerebral infarct volume of $29.60 \% \pm 5.16 \%$. In addition, neurologic deficits were assessed by scoring specific behaviors. As shown in Fig. 2C, the neurologic deficit score of the I/R group $(2.87 \pm 0.41)$ was significantly higher than the score of the control group. Following pretreatment with PNE (100 mg kg $\left.\mathrm{mg}^{-1}\right)$, significant decreases in neurologic deficit scores $(1.90 \pm 0.22)$ were observed compared with the I/R group $(P<$ $0.001)$, which indicates that PNE improved neurologic deficits caused by cerebral I/R. These results robustly demonstrate that PNE exerts a remarkable neuroprotective effect from cerebral I/R injury in rats.

The Role of Gut Microbiota in Neuroprotective Effects of PNE. PGF model rats were used to investigate the role of gut microbiota in the neuroprotective effects of PNE. As shown in Fig. 2D, cerebral infarcts were prominent after establishing the I/R model in PGF rats. Cerebral infarct volume was $36.81 \% \pm 4.61 \%$ in the $\mathrm{PGF}+\mathrm{I} / \mathrm{R}$ group (Fig. $2 \mathrm{E}$ ). In the PNE treatment group, infarct size was reduced to $29.60 \% \pm$ $5.16 \%$. In addition, the neurologic deficit score of the PGF $+\mathrm{I} / \mathrm{R}$ group was slightly higher than that of the PNE treatment group. The neurologic deficit scores of the PGF $+\mathrm{I} / \mathrm{R}$ and PNE treatment groups were $2.79 \pm$ 0.53 and $2.25 \pm 0.25$, respectively (Fig. $2 F$ ). However, comparisons of cerebral infarct volumes and neurologic deficit scores between PGF + $\mathrm{I} / \mathrm{R}$ and $\mathrm{I} / \mathrm{R}$ rats indicated that the neuroprotective effects of PNE in the $\mathrm{PGF}+\mathrm{I} / \mathrm{R}$ rats were much lower than in normal I/R rats. These results suggest that gut microbiota play an important role in neuroprotective effects of PNE.

I/R-induced brain injury is closely associated with enhanced inflammatory responses and lower BDNF levels. Therefore, proinflammatory cytokine and BDNF levels in control, I/R, I/R + PNE, PGF, PGF $+\mathrm{I} / \mathrm{R}$, and $\mathrm{PGF}+\mathrm{I} / \mathrm{R}+\mathrm{PNE}$ groups were determined to verify further the role of gut microbiota in the neuroprotective effects of PNE. As shown in Fig. 2, G and H, levels of IL- $1 \beta$ and IL-6 in the hippocampus of the I/R group were significantly increased compared with the control group (one-way ANOVA, $P<0.01$ ). After pretreatment with PNE, levels of IL- $1 \beta$ and IL-6 were significantly decreased (one-way ANOVA, $P<$ 0.01 ). IL- $1 \beta$ and IL-6 levels in the PGF group were similar to those in the control group, and no significant difference was found between the I/R and PGF + I/R groups. In the PGF + I/R + PNE group, IL- $1 \beta$ and IL-6 levels were lower than those in the PGF $+\mathrm{I} / \mathrm{R}$ group. However, IL- $1 \beta$ levels in the PGF $+\mathrm{I} / \mathrm{R}+\mathrm{PNE}$ group were markedly higher than in the $\mathrm{I} / \mathrm{R}+\mathrm{PNE}$ group (one-way ANOVA, $P<0.05$ ), and IL-6 levels in the $\mathrm{PGF}+\mathrm{I} / \mathrm{R}+\mathrm{PNE}$ group were also higher than in the $\mathrm{I} / \mathrm{R}+\mathrm{PNE}$ group to some extent. These results demonstrate that gut microbiota greatly influence regulation of proinflammatory cytokines by PNE in I/R rats.

BDNF is a member of the nerve growth factor family and is involved in a plethora of functions within the CNS, including neuronal survival and differentiation (Bjorkholm and Monteggia, 2016). Thus, regulation
A

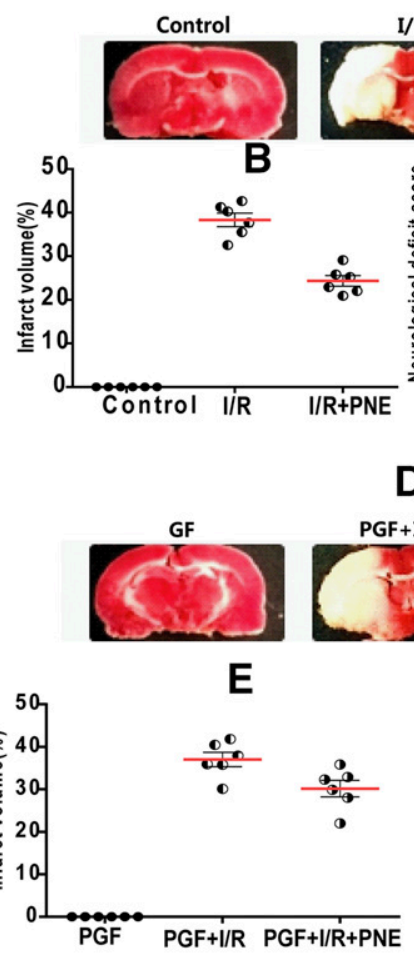

I/R

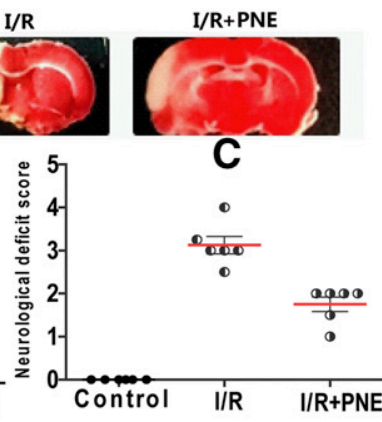

D
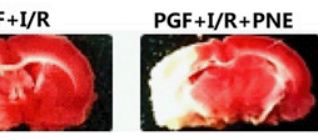

$\mathbf{F}$

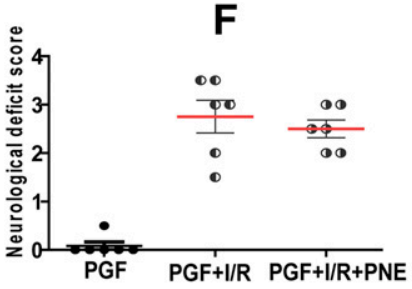

G

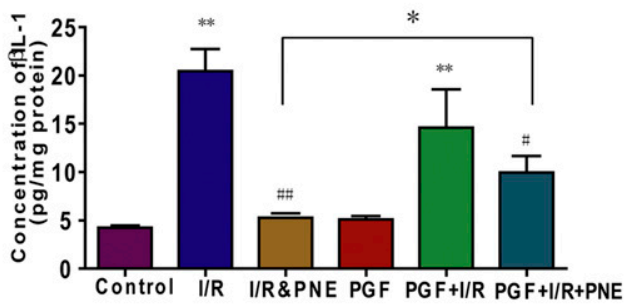

H

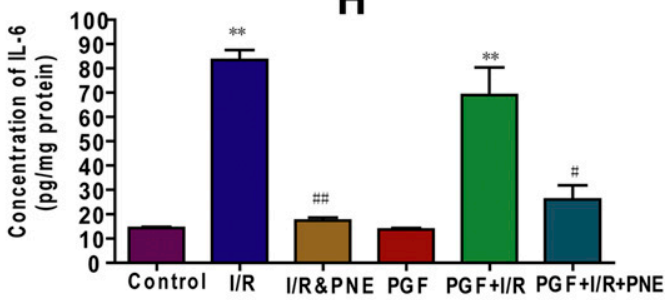

I

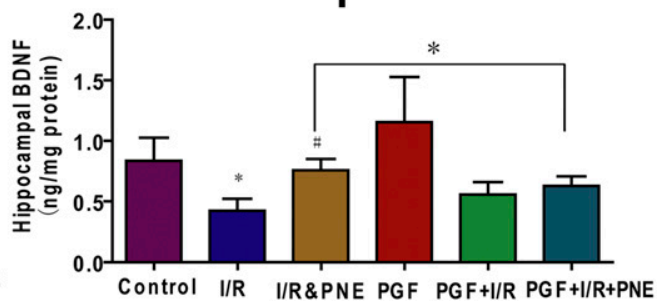

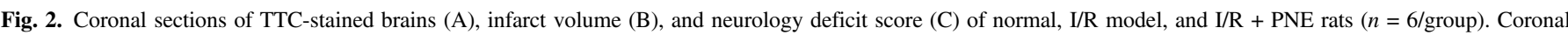

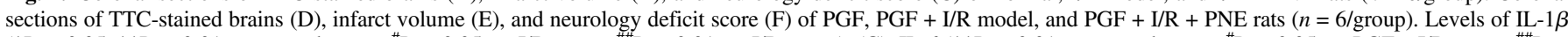

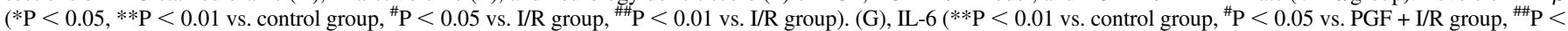
0.01 vs. I/R group). (H), and BDNF ( ${ }^{*} \mathrm{P}<0.05$ vs. control group, ${ }^{\#} \mathrm{P}<0.05$ vs. I/R group). (I) in rat hippocampus $(n=6 /$ group). 
of BDNF expression was assessed to investigate its role in the neuroprotective effects of PNE. As shown in Fig. 2I, levels of BDNF were significantly decreased in I/R rats compared with the control group, whereas PNE treatment could significantly attenuate this tendency (one-way ANOVA, $P<0.05$ ). However, PNE could not upregulate expression of BDNF in $\mathrm{PGF}+\mathrm{I} / \mathrm{R}$ rats, and expression of BDNF in the $\mathrm{I} / \mathrm{R}+\mathrm{PNE}$ group was significantly higher than in the $\mathrm{PGF}+\mathrm{I} / \mathrm{R}+\mathrm{PNE}$ group. Thus, these results suggest that gut microbiota influence regulation of BDNF expression during PNE-induced protective effects on the brain after cerebral I/R.

PNE Regulation of Gut Microbiota Populations. Intestinal bacterial community structures were characterized on the basis of the 16S-rRNA-amplicon Illumina sequencing to investigate intestinal flora associated with protective effects of PNE in the brain. The taxon abundance of each sample was divided into phylum, class, order, family, and genera levels. First, 58414, 61311, 60633, 62915, 66084, and $6587216 \mathrm{~S}$ rRNA valid-sequence-reads were obtained from samples in the control, I/R, I/R + PNE, PGF, PGF + I/R, and PGF + $\mathrm{I} / \mathrm{R}+\mathrm{PNE}$ groups, respectively (Supplemental Fig. 1). This indicated that the $16 \mathrm{~S}$ rRNA gene sequence database of each group was abundant enough to capture most microbial diversity information. To clearly highlight differences in bacterial community structures among the groups, stacked column charts of the dominant bacterial genera were constructed on the basis of the top 10 most abundant gut microbes in each group using the QIIME toolkit (Quantitative Insights Into Microbial Ecology; www.qiime.org) (Supplemental Fig. 2). As shown in Supplemental Fig. 2A, Bacteroidetes, Firmicutes, and Proteobacteria were identified as the major phyla of the rat GI bacterial community. The relative abundance of Proteobacteria and Firmicutes in the I/R group was much lower than in the control group, and pretreatment with PNE could significantly reduce changes in intestinal bacterial community structure caused by I/R surgery. Likewise, large differences in bacterial class and order were observed between the control and I/R groups. Furthermore, PNE could shift the gut microbiota populations of the I/R group to be more like those of the control group (Supplemental Fig. 2, B and C). For PGF rats, the gut microbiota populations were dominated by Blautia, Erysipelatoclostridium, Alistipes, and Paraprevotella, which belong to the Firmicutes and Bacteroidetes phyla. A comparison of gut microbiota populations in the PGF, PGF $+\mathrm{I} / \mathrm{R}$, and PGF $+\mathrm{I} / \mathrm{R}+\mathrm{PNE}$ groups showed that both I/R surgery and pretreatment with PNE could change intestinal bacterial community structures of PGF rats, although the magnitude of the changes was weakened compared with that in normal rats.

According to previous reports, L.R, L.B, L.H, and B.L could play crucial roles in the bidirectional gut-brain axis by modulating stressinduced behavioral deficits, immune changes, and gut dysbiosis (Bravo et al., 2011; Barrett et al., 2012; Borrelli et al., 2016; Bharwani et al., 2017). To explore further the types of intestinal bacteria that mediate brain protection, the relative abundances of L.R, L.B, L.H, and B.L in control, I/R, and I/R + PNE groups were measured using real-time PCR. As shown in Fig. 3A, the relative abundances of these four species of bacteria were downregulated after I/R surgery, and pretreatment with PNE could significantly upregulate the relative abundance of L.B (oneway ANOVA, $P<0.05$ ) and B.L (one-way ANOVA, $P<0.01$ ) in $\mathrm{I} / \mathrm{R}$ rats. Given that PNE regulation of B.L was much higher than that of other bacteria, we compared the population of B.L in control, I/R, I/R + PNE, $\mathrm{PGF}, \mathrm{PGF}+\mathrm{I} / \mathrm{R}$, and PGF $+\mathrm{I} / \mathrm{R}+\mathrm{PNE}$ groups. As shown in Fig. $3 \mathrm{~B}$, the population of B.L in PGF, PGF $+\mathrm{I} / \mathrm{R}$, and $\mathrm{PGF}+\mathrm{I} / \mathrm{R}+\mathrm{PNE}$ groups was
A

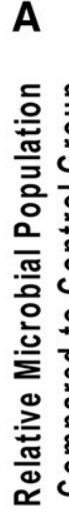

B
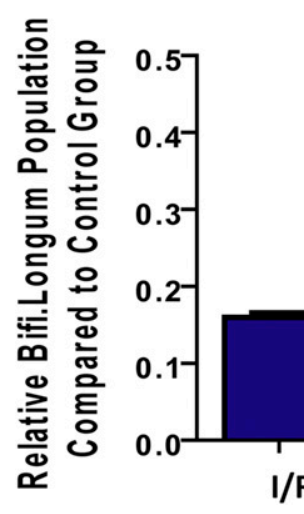

B
I/R

I/R+PNE
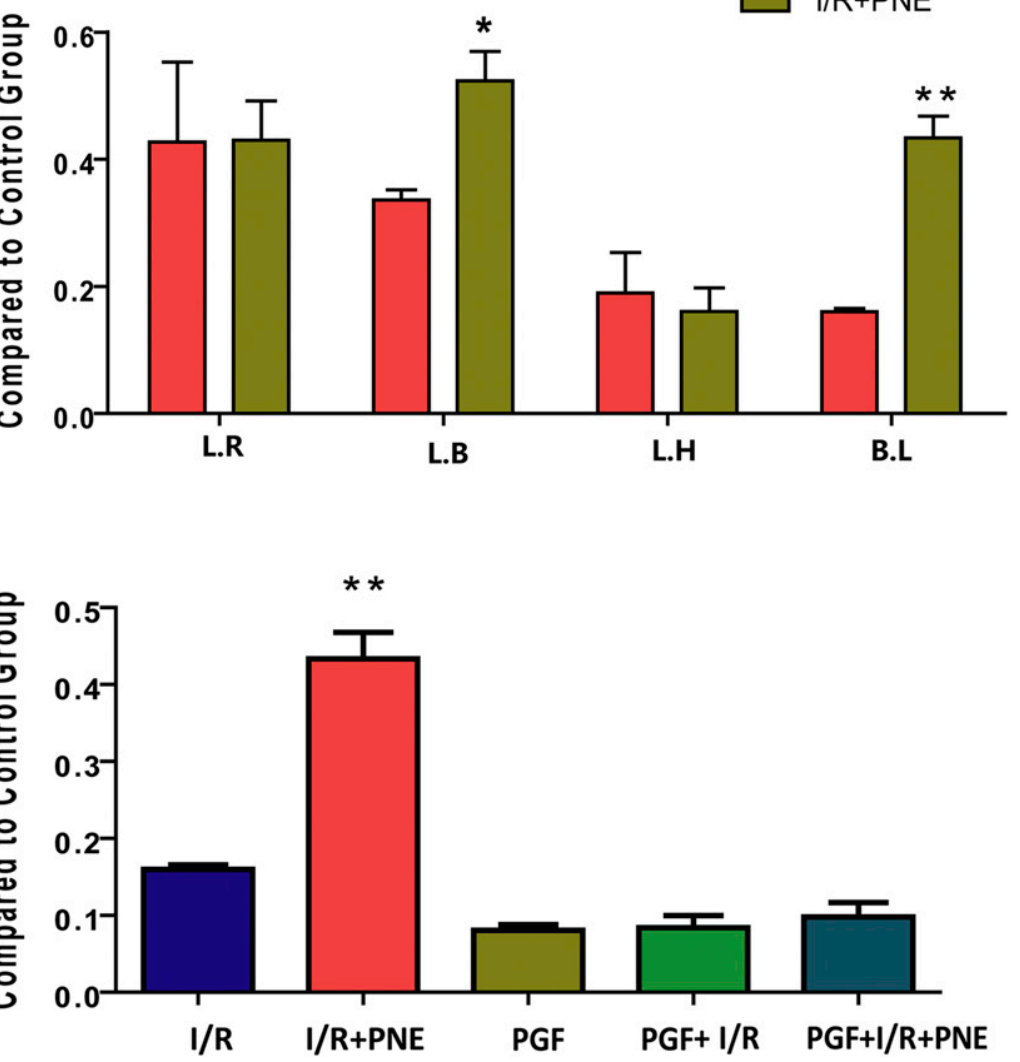

Fig. 3. Relative abundance of the dominant bacteria in the rat intestinal microflora ( $n=6 /$ group). (A) The relative abundances of L.R, L.B, L.H, and B.L in control, I/R, and I/R + PNE rat intestinal microflora. (B) The relative abundance of B.L in control, I/R, I/R + PNE, PGF, PGF + I/R, and PGF + I/R + PNE rats. $(* \mathrm{P}<0.05, * * \mathrm{P}<0.01)$. 
much lower than that in control, I/R, and I/R + PNE groups. PNE could significantly prevent the decrease in B.L caused by I/R surgery, but it did not have a significant effect on B.L in PGF and PGF + I/R rats.

Neuroprotective Effects of B.L for I/R Model Rats. According to the results above, the decrease in relative abundance of B.L in I/R rats could be prevented by pretreating with PNE for 7 consecutive days. Thus, we investigated whether B.L exerted neuroprotective effects. Rats were pretreated with $1 \times 10^{9} \mathrm{CFU}$ of B.L per day for 15 consecutive days before I/R surgery. TTC staining was used to examine cerebral infarcts 24 hours after I/R surgery. Figure 4A shows representative images of coronal sections from the sham-operated (control), I/R, and B. L-treated groups. Cerebral infarcts were obvious after establishing the I/R model. Infarct size in the B.L treatment group was significantly decreased compared with that in the I/R group; cerebral infarct volume decreased from $36.0 \% \pm 4.3 \%$ to $25.0 \% \pm 9.2 \%$ (Fig. 4B). In addition, after pretreatment with B.L, significant decreases in neurologic deficit scores $(1.96 \pm 0.27)$ were observed compared with the I/R group (2.83 \pm 0.47 ), which indicated that B.L improved neurologic deficits caused by cerebral I/R (Fig. 4C). Furthermore, B.L could significantly downregulate levels of proinflammatory cytokines (IL- $1 \beta$, IL-6, and TNF- $\alpha$ ) in I/R rats (Fig. 4, D-F). Likewise, I/R surgery significantly decreased levels of BDNF in the rat hippocampus (Fig. 4G), and B.L treatment could efficiently attenuate the downregulation of BDNF caused by I/R surgery. These results suggest that colonization by B.L can exert neuroprotective effects in I/R rats.

Effects of PNE and B.L on the Expression of GABA Receptor in the Rat Hippocampus. Recent studies have suggested that in addition to playing an important role in maintaining functional homeostasis in the brain, GABA receptors are vulnerable to pathologic factors, especially during ischemia (Fiorentino et al., 2009; Cheng et al., 2010; Fu et al., 2015; Nasrallah et al., 2017). To elucidate further the mechanism underlying therapeutic effects of PNE during I/R, effects of PNE and B.L on expression of GABA receptors were investigated. Expression levels of GABA-A receptors $(\alpha 2, \beta 2, \gamma 2)$ and GABA-B receptors (R1b, R2) in the hippocampus of normal and PGF rats were assessed using reverse transcription PCR. For normal rats, expression level ratios of GABA-A receptors $(\alpha 2, \beta 2, \gamma 2)$ and GABA-B receptors $(\mathrm{R} 1 \mathrm{~b}, \mathrm{R} 2)$ in the hippocampus from $\mathrm{I} / \mathrm{R}$ and control groups were less than 1 , which indicated that $\mathrm{I} / \mathrm{R}$ surgery could decrease expression levels of GABA receptors (Fig. 5A, one-way ANOVA, $P<0.05$ ). PNE pretreatment could significantly attenuate the decrease in expression levels of GABA receptors in the I/R group. For PGF rats, I/R surgery could also distinctly decrease expression of GABA receptors in the hippocampus. However, the ability of PNE to regulate expression of GABA receptors in PGF rats

A

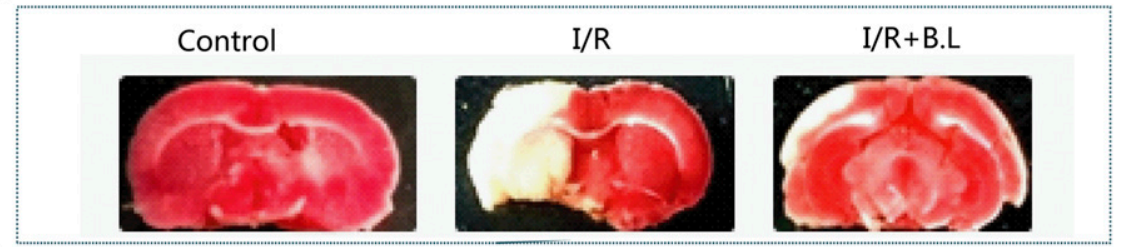

B

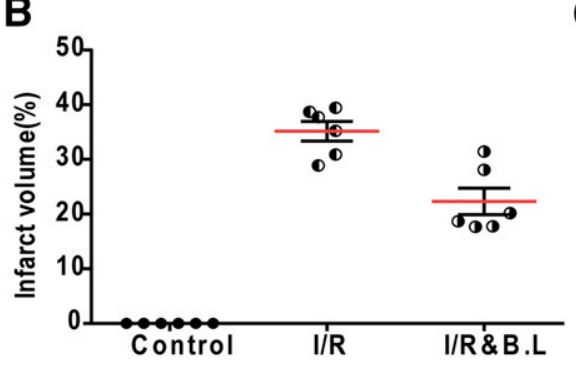

D
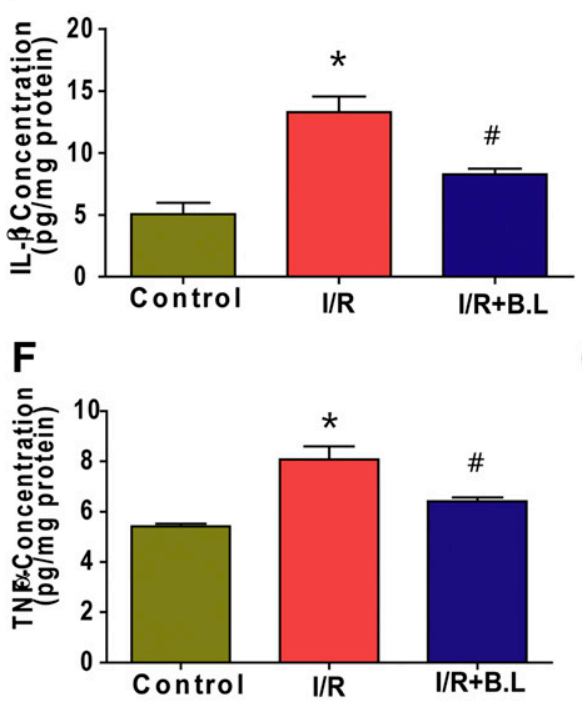

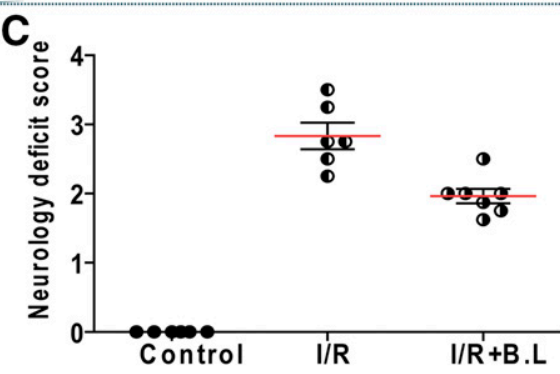

$\mathrm{E}$

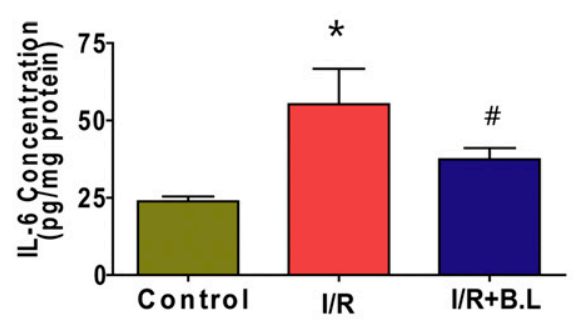

G

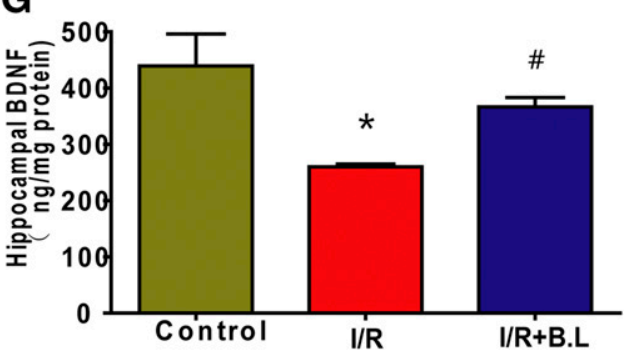

Fig. 4. The neuroprotective effects of B.L $(n=$ 6/group). (A) Coronal sections of TTC-stained brains and (B) infarct volume. (C) Neurology deficit score, (D) IL-1 $\beta$, (E) IL-6, (F) TNF- $\alpha$, (G) BDNF. $\left({ }^{*} \mathrm{P}<0.05\right.$ vs. control group, ${ }^{\#} \mathrm{P}<0.05$ vs. I/R group). 
A

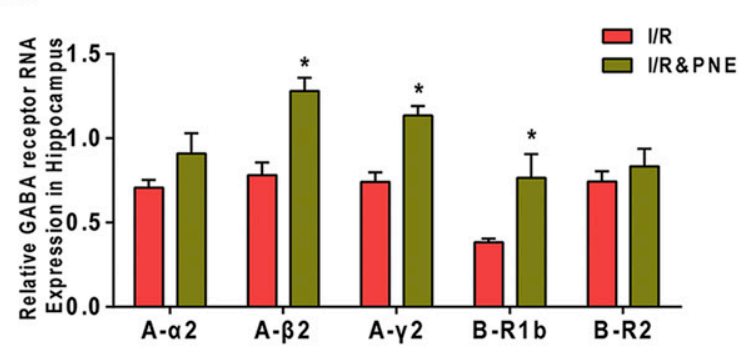

C

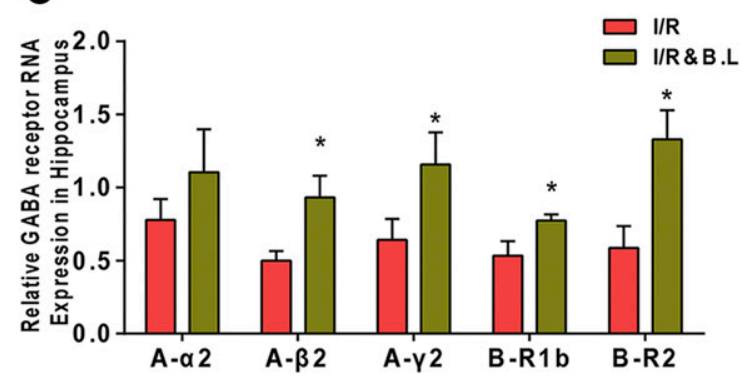

B

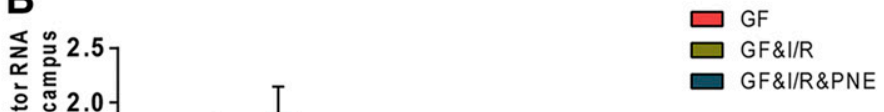

GF\&l/R GF\&I/R\&PNE

D

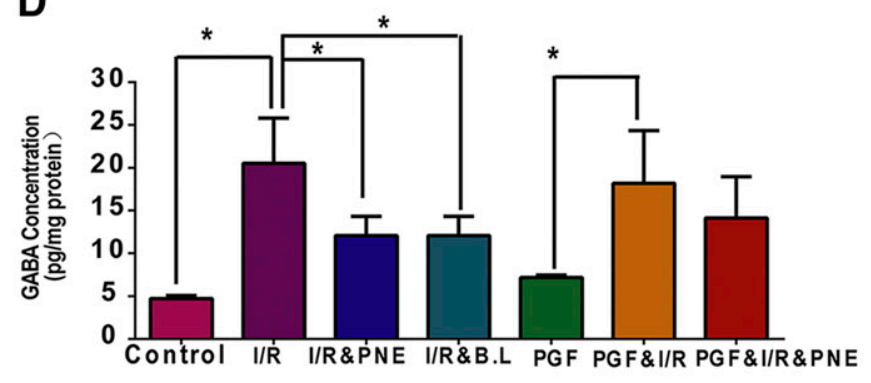

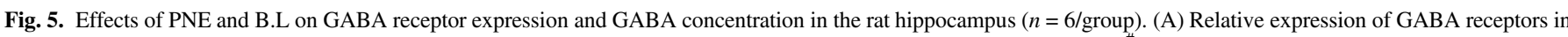

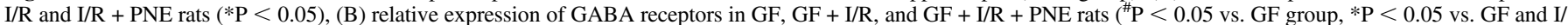
$\mathrm{R}$ group), (C) relative expression of GABA receptors in $\mathrm{I} / \mathrm{R}$ and $\mathrm{I} / \mathrm{R}+\mathrm{B}$.L rats $* \mathrm{P}<0.05$, (D) GABA concentrations in rat hippocampus $* \mathrm{P}<0.05$.

was much lower than in normal rats (Fig. 5B, one-way ANOVA, $P<$ 0.05). These results suggest that upregulation of GABA receptors by PNE was attenuated by antibiotic treatment owing to a shift in gut microbiota populations. We also examined whether B.L could regulate GABA receptors in the hippocampus. Expression level ratios of GABA-A receptors $(\alpha 2, \beta 2, \gamma 2)$ and GABA-B receptors $(\mathrm{R} 1 b, \mathrm{R} 2)$ in the B.Lcolonized group were much higher than in the I/R group (Fig. 5C, one-way ANOVA, $P<0.05$ ). Thus, these results suggested that B.L could prevent decreased expression levels of GABA receptors in the I/R group.

Given that both PNE and B.L could enhance expression of GABA receptors in $\mathrm{I} / \mathrm{R}$ rats, GABA concentrations in the hippocampus were determined to investigate further PNE regulation of the GABAergic system. As shown in Fig. 5D, I/R surgery led to an increase in GABA levels, and pretreating with PNE could significantly decrease GABA concentrations in the I/R group. Likewise, colonization with B.L could prevent an increase in GABA levels caused by I/R surgery. For PGF rats, I/R surgery could also enhance GABA levels. However, the effect of PNE on GABA levels in $\mathrm{PGF}+\mathrm{I} / \mathrm{R}$ rats was not as significant as in normal $\mathrm{I} / \mathrm{R}$ rats.

Effects of GABA-A and GABA-B Receptor Antagonists on Neuroprotective Effects of B.L. Given that both PNE and B.L could upregulate expression of GABA receptors in the hippocampus of I/R rats (Fig. 5A and 5C), we investigated whether GABA-A or GABA-B receptors mediate the neuroprotective effects. A GABA-A receptor antagonist (bicuculline, Bic) or GABA-B receptor antagonist (saclofen, Sac) was used to inhibit GABA-A or GABA-B receptor activities, respectively. As shown in Fig. 6A, neuroprotective effects of B.L were significantly attenuated when the bacteria were coadministered with the GABA-B receptor antagonist Sac. In contrast, the GABA-A receptor antagonist Bic had no significant effect. The cerebral infarct volumes of rats in the $\mathrm{I} / \mathrm{R}, \mathrm{I} / \mathrm{R}+\mathrm{B} . \mathrm{L}, \mathrm{I} / \mathrm{R}+\mathrm{B} . \mathrm{L}+\mathrm{Bic}$, and $\mathrm{I} / \mathrm{R}+\mathrm{B} . \mathrm{L}+\mathrm{Sac}$ groups were $36.0 \% \pm 4.3 \%, 25.0 \% \pm 9.2 \%, 26.2 \% \pm 3.1 \%$, and $34.9 \% \pm 5.1 \%$, respectively (Fig. 6B). The neurologic deficit scores of rats in the I/R, I/R + B.L, I/R + B.L + Bic, and I/R + B.L + Sac groups were $2.83 \pm 0.46,1.96 \pm$ $0.28,2.10 \pm 0.77$, and $2.80 \pm 0.25$, respectively (Fig. 6C). No obvious differences in expression of proinflammatory cytokines IL-1 $\beta$, IL-6, and
TNF- $\alpha$ were found between the I/R + B.L and I/R + B.L + Bic groups. When saclofen was coadministered with B.L, the neuroprotective effects of B.L were dramatically attenuated, and expression levels of proinflammatory cytokines IL- $1 \beta, \mathrm{IL}-6,7$ and TNF- $\alpha$ in the I/R + B.L + Sac group were much higher than in the I/R + B.L and I/R + B.L + Bic groups (Fig. 6, D-F). These results strongly suggested that upregulation of GABA-B receptors might be a key factor in mediating neuroprotective effects of B.L and PNE.

The influence of PNE and B.L on the GABA-B receptors (R1, R2) was further verified on the basis of Western blotting analysis. As shown Fig. 7, the I/R surgery led to a decrease in the expression of GABA-B R1 receptor, and the expression of GABA-B R2 receptor in I/R group was significantly lower than that of the control group. Importantly, pretreating with PNE could upregulate the expression of GABA-B R1 and GABA-B R2 receptors in the hippocampus of I/R model rats. Likewise, colonization with B.L could prevent a decrease in the expression of GABA-B R1 and GABA-B R2 receptors caused by I/R surgery.

Attenuating Effects of GABA-B Receptor Antagonists on Efficacy of PNE. Given that neuroprotective effects of B.L could be greatly attenuated by a GABA-B receptor antagonist, we examined whether a GABA-B receptor antagonist also attenuated neuroprotective effects of PNE. The GABA-B receptor antagonist, saclofen, was coadministered with PNE to confirm that neuroprotective effects of PNE were mediated by B.L and the GABAergic system. As shown in Fig. 8, A-C, neuroprotective effects of PNE were drastically attenuated by the GABA-B receptor antagonist. The cerebral infarct volumes of rats in the I/R + PNE and I/R + $\mathrm{PNE}+$ Sac groups were $25.0 \% \pm 9.2 \%$ and $33.4 \% \pm 3.0 \%$, respectively, and neurology deficit scores of rats in the I/R + PNE and I/R + PNE + Sac groups were $1.68 \pm 0.41$ and $2.54 \pm 0.29$, respectively. Likewise, levels of proinflammatory cytokines IL- $1 \beta$, IL-6, and TNF- $\alpha$ in the I/R + PNE + Sac group were significantly higher than in the $\mathrm{I} / \mathrm{R}+\mathrm{PNE}$ group.

\section{Discussion}

This study demonstrates a specific, previously unreported neuroprotective mechanism of PNE on the basis of the microbiome-gut-brain 
A

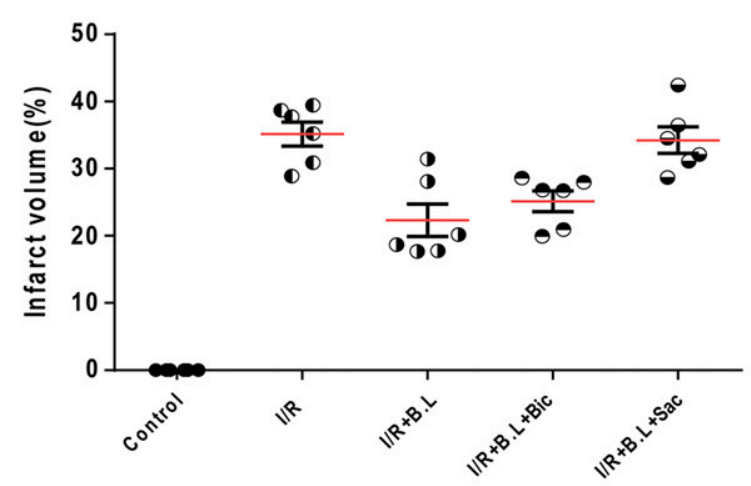

C

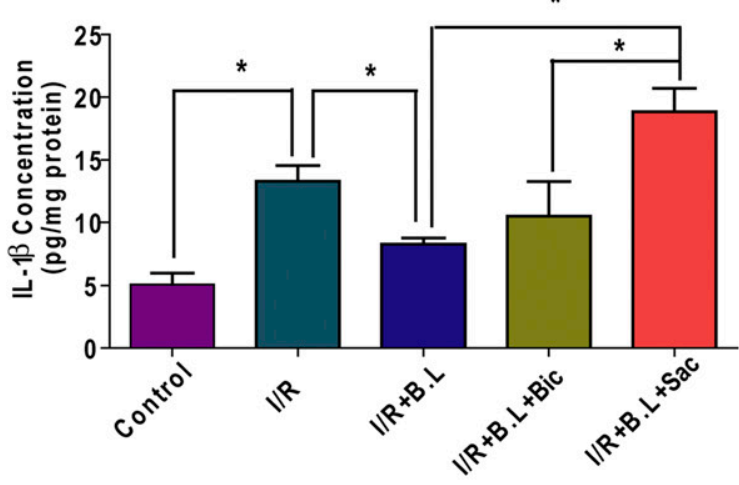

$\mathbf{E}$

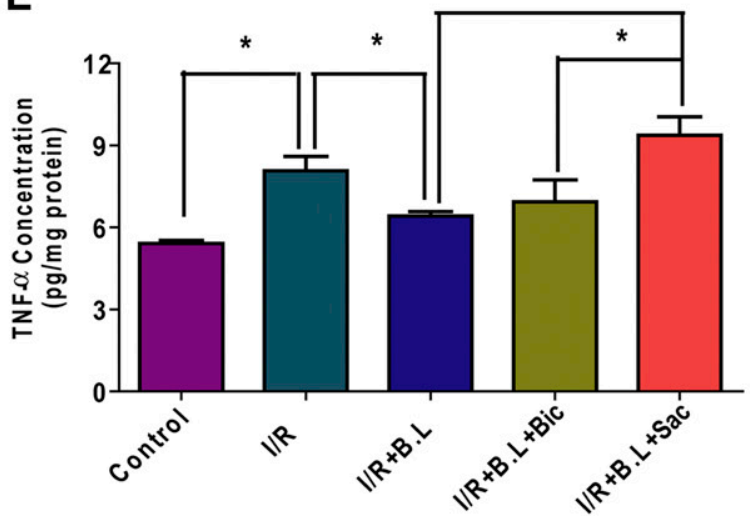

B

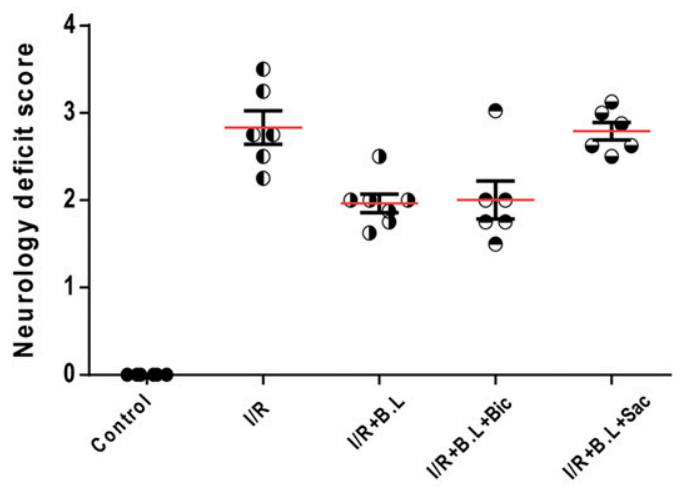

D

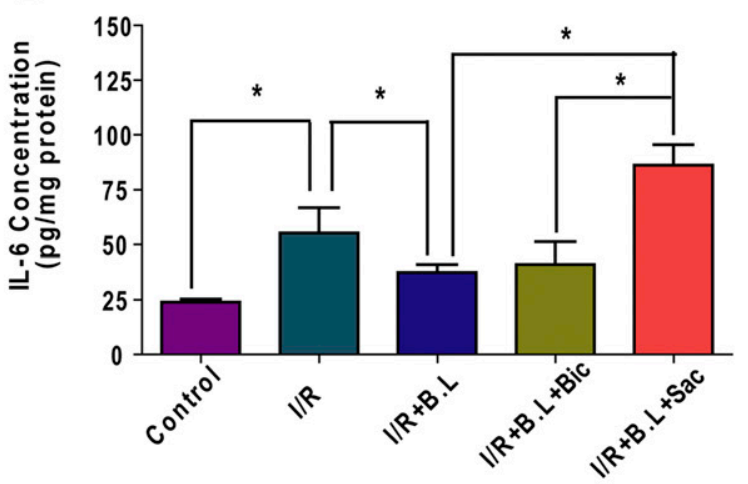

$\mathbf{F}$

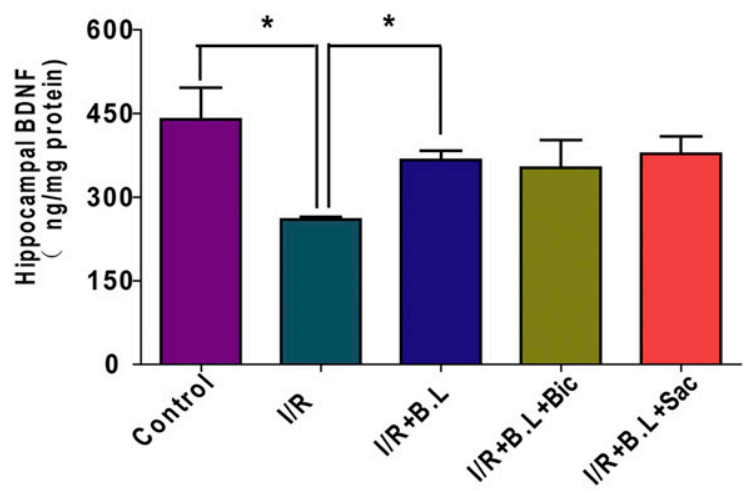

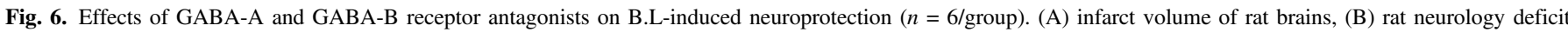
score, (C) IL-1 $\beta$ levels, (D) IL-6 levels, (E) TNF- $\alpha$ levels, and (F) BDNF levels.

axis. In our previous studies, the optimal dose of PNE was screened by comparing the neuroprotective effects of PNE under different doses $(20,50,100$, and $250 \mathrm{mg} / \mathrm{kg})$. The results revealed that the neuroprotective effects of PNE were dose-dependent within the 20 $100 \mathrm{mg} / \mathrm{kg}$ dosage range, and the efficiency apparently increased with the increasing dose. At 100 and $250 \mathrm{mg} / \mathrm{kg}$ doses, there was no significant difference in the neuroprotective effects of PNE. Hence, $100 \mathrm{mg} / \mathrm{kg}$ of PNE was used to investigate the neuroprotective effect of PNE in the present study. We found that pretreatment with PNE $(100 \mathrm{mg} / \mathrm{kg})$ for 7 consecutive days before cerebral I/R could significantly decrease levels of proinflammatory cytokines, reduce the volume of brain infarcts, attenuate neurologic deficits, and maintain BDNF levels in the hippocampus even though exposure to each component of PNE was extremely low in the brains of normal and $I / R$ rats. For herbal saponins, oral bioavailability is often limited owing to low membrane permeability, high molecular weight, fast clearance in the GI tract, and efflux transporters (Hao et al., 2016). Clinically, some commercial PNE formulations cure cerebral diseases mainly after intravenous administration, as exemplified by the Xue-Sai-Tong injection. However, inconvenient medication and adverse reactions caused by injection have limited the application of PNE to a large extent (Guo et al., 2014). In the present study, we found that cerebral concentrations of notoginsenoside monomers in the I/R group were higher than in the control group. This phenomenon could be explained by increased permeability of a compromised blood-brain barrier after ischemic brain insult. However, most of the notoginsenoside monomers were undetectable at 10 hours after oral administration, which indicated that neuroprotective effects of PNE may not be mediated directly by cerebral notoginsenoside monomers. The contradiction between 


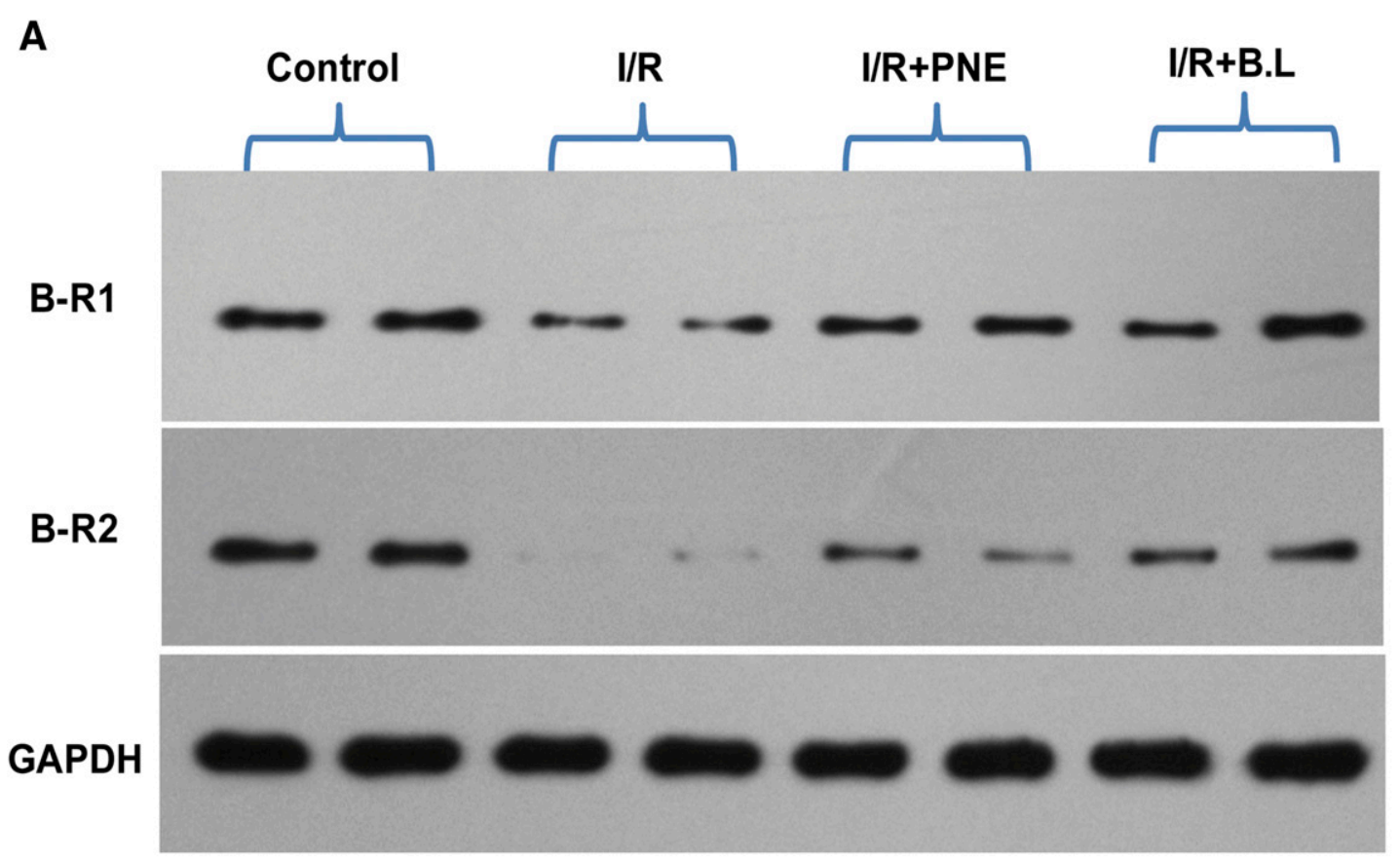

B

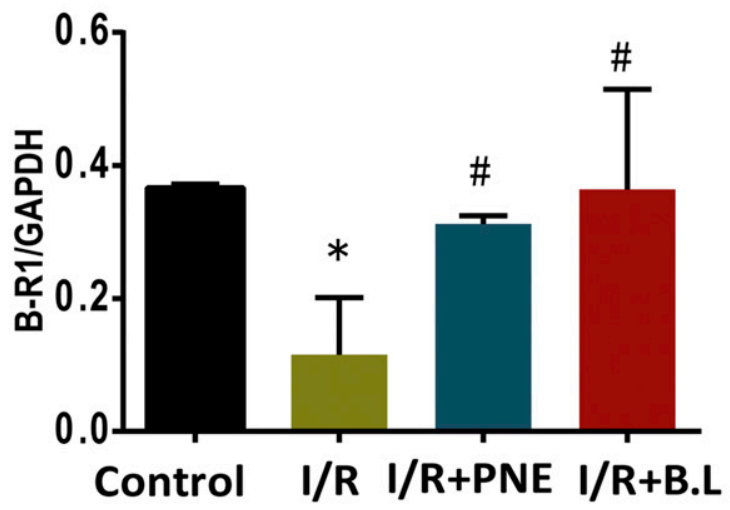

C

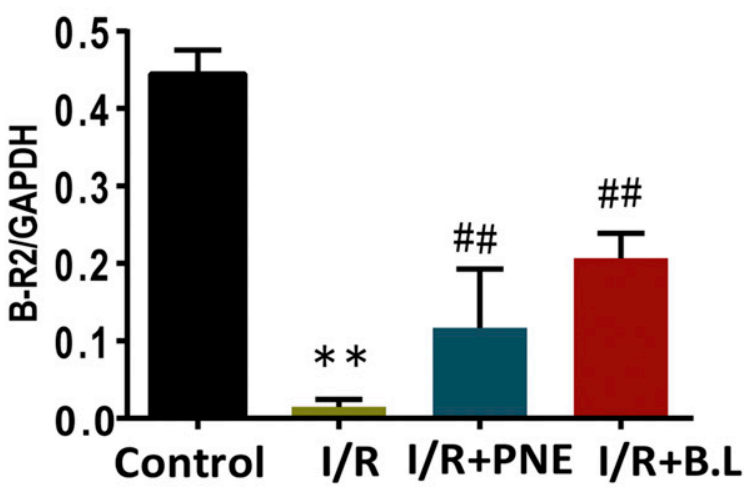

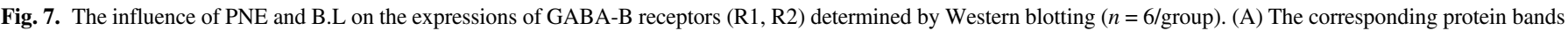

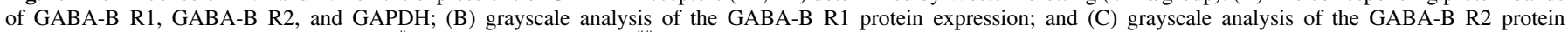
expression. ( ${ }^{*} \mathrm{P}<0.05$ vs. control group, ${ }^{\#} \mathrm{P}<0.05$ vs. I/R group, ${ }^{\# \#} \mathrm{P}<0.01$ vs. I/R group).

pharmacokinetic and pharmacodynamic profiles of PNE led us to hypothesize that PNE may exert neuroprotective effects indirectly by acting on peripheral targets.

Recent studies have revealed that intestinal microflora play a role in development of cells and tissues, affect physiologic, nutritional, and immunologic processes, as well as directly and indirectly alter pharmacokinetics and pharmacological activities of drugs, especially those that are orally administered (Wallace et al., 2010; Haiser and Turnbaugh, 2012). Given that the residence time of notoginsenosides in the intestine is significantly longer than in other sites (Liu et al., 2009), interactions between notoginsenosides and intestinal microflora are worth exploring. In our previous study, we found that intestinal microflora greatly affect metabolism of notoginsenosides. For example, Proteobacteria can affect deglycosylation metabolism of notoginsenosides by regulating activities of glycosidases, and upregulation of Bacteroidetes can promote redox metabolism of notoginsenosides in intestinal microflora (Xiao et al., 2016). In the present study, a PGF rat model was established to investigate the role of gut microbiota in neuroprotective effects of PNE. We found that neuroprotective effects of
$\mathrm{PNE}$ in $\mathrm{PGF}+\mathrm{I} / \mathrm{R}$ rats were much lower than in normal $\mathrm{I} / \mathrm{R}$ rats. Thus, gut microbiota play an important role in mediating neuroprotective effects of PNE. The influence of PNE on the gut microbiota community was investigated to clarify the bidirectional regulation of intestinal flora and notoginsenosides. The results revealed that $\mathrm{I} / \mathrm{R}$ surgery could dramatically alter the structure of the gut microbiota community, and cerebral I/R-induced changes in gut microbiota could be partially prevented by pretreating with PNE for 7 consecutive days. In recent years, there has been a growing interest in targeting the gut microbiome for a beneficial impact on behaviors related to psychiatric disorders, including anxiety, depression, autism spectrum disorder, obsessivecompulsive disorder, and memory impairment (Wang et al., 2016). To date, multiple probiotic bacteria with psychotropic potential, including strains of the genera Lactobacillus (Messaoudi et al., 2011a), Bifidobacterium (Messaoudi et al., 2011b), and Enterococcus (Divyashri et al., 2015), have been identified as drug action targets or potential therapeutic psychobiotics. For example, L.R is a very promising potential psychobiotic with demonstrated pharmacological activity in mice and humans (Borrelli et al., 2016). Oral administration of L.B can 
A

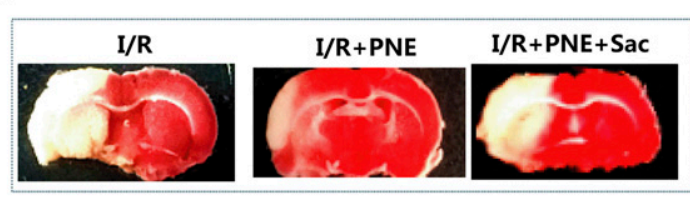

B
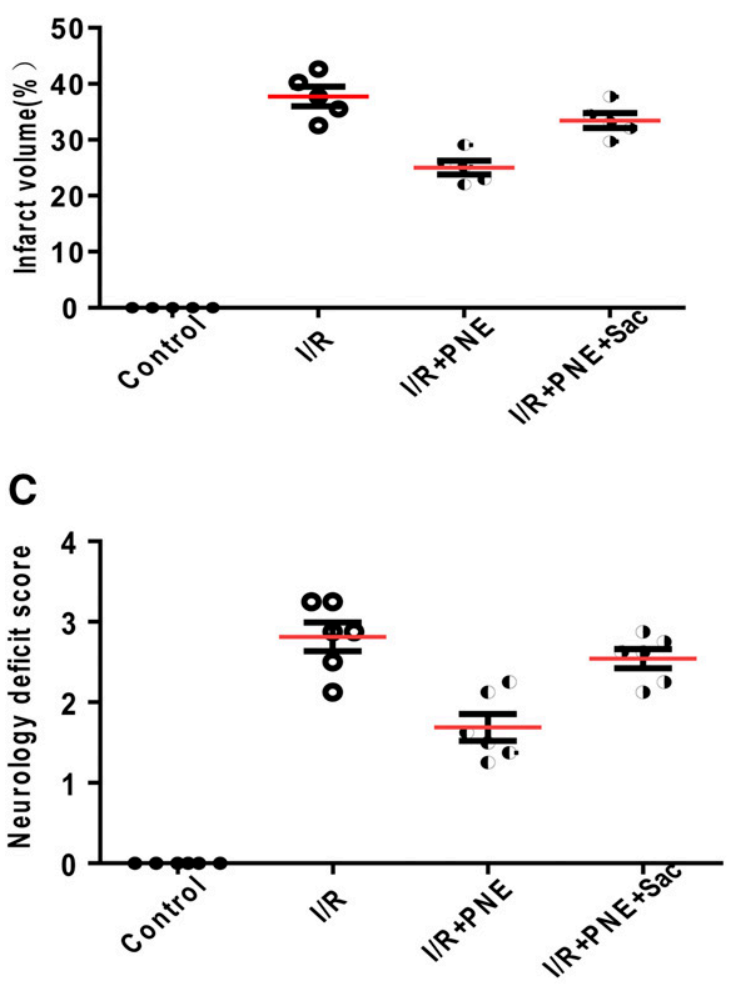

D

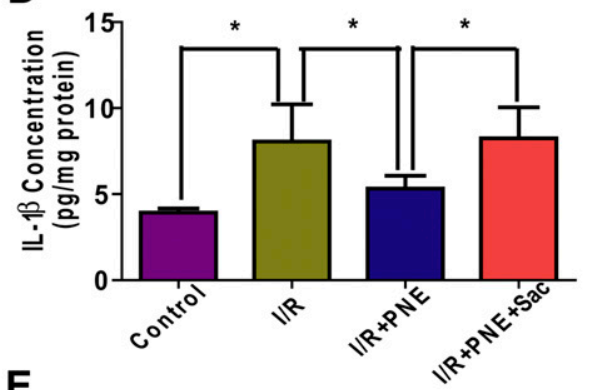

$\mathbf{E}$

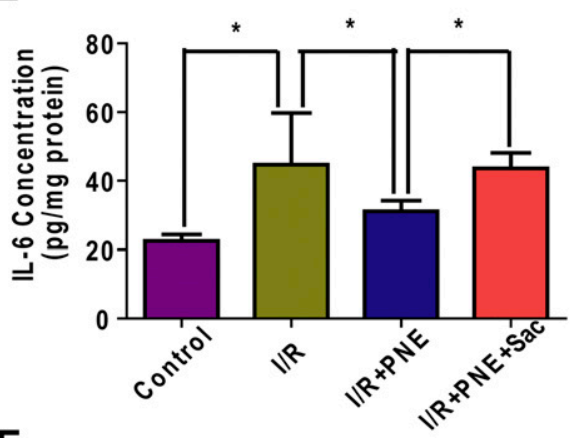

$\mathbf{F}$

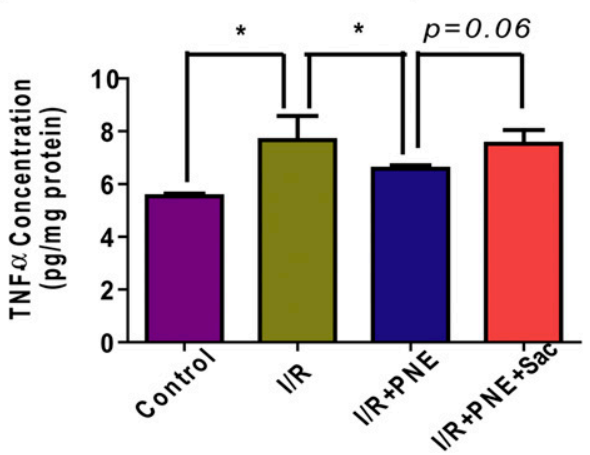

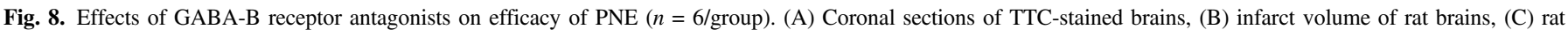
neurology deficit score, (D) IL- $1 \beta$ levels, (E) IL-6 levels, (F) TNF- $\alpha$ levels. $(* \mathrm{P}<0.05$ ).

significantly inhibit lipopolysaccharide production and P16 expression in lipopolysaccharides-stimulated macrophages (Jeong et al., 2016). In addition, a single strain of B.L or L.H can improve anxiety-like behaviors in immune-deficient mice and chronically restrained rats (Ohland et al., 2013; Savignac et al., 2014). In the present study, the relative abundances of CNS disease therapy-related strains (L.R, L.B, L. $\mathrm{H}$, and B.L) were measured to investigate the therapeutic target and mechanism of PNE for cerebral I/R. The results demonstrated that the relative abundances of all four species of bacteria were downregulated after I/R surgery, and pretreating with PNE could significantly upregulate the relative abundance of B.L. We chose B.L as a potential therapeutic bacterial species for cerebral I/R since it had been reported to improve anxiety-like behavior and normalize levels of hippocampal BDNF in mice (Bercik et al., 2011). After colonizing B.L $\left(1 \times 10^{9} \mathrm{CFU}\right)$ by gavage 15 days before I/R surgery in rats, the I/R surgery-induced effects on infarct size, neurologic deficit score, proinflammatory cytokines (IL-1 $\beta$, IL-6, and TNF- $\alpha$ ), and BDNF could be prevented.

GABA is the primary inhibitory neurotransmitter in the mammalian CNS and acts on GABA-A and GABA-B receptors (Vollenweider et al., 2006). Numerous studies have demonstrated that GABAergic neurotransmission in the hippocampus is closely related to modulation of behavior and memory, and GABA receptors play a pivotal role in treatment of transient focal cerebral ischemia (Vollenweider et al., 2006;
Wang et al., 2007; Han et al., 2008; Cheng et al., 2010). To bridge the gap between PNE and GABA receptors, the influence of B.L on expression of GABA receptors was investigated. The results revealed that pretreatment with B.L could upregulate expression of GABA-A and GABA-B receptors in the rat hippocampus. In addition, PNE pretreatment could significantly upregulate expression levels of GABA receptors in the hippocampus of rats in the I/R group. To confirm the role of B.L in PNE regulation of GABA receptors, we compared expression of GABA receptors in the hippocampus of normal and PGF rats. The results suggest that $\mathrm{I} / \mathrm{R}$ surgery can decrease expression of GABA receptors in the hippocampus of PGF rats and that the ability of PNE to regulate expression of GABA receptors is much lower in PGF rats compared with normal rats.

Numerous GABAergic neurons are located in the hippocampal dentate gyrus, and GABA-A and GABA-B receptors in this region are the two major classes of receptors that regulate synaptic plasticity and learning and memory processes (Shahidi et al., 2008). The GABA-A receptor, which is a ligand-gated ion channel, is a pentameric structure assembled from 5 of the 19 known protein subunits, including $\alpha 1-6$, $\beta 1-3, \gamma 1-3, \delta, \varepsilon, \pi, \rho 1-3$, and $\theta$ (McGinnity et al., 2017). Experiments in animals suggest that alteration of GABA-A receptor-mediated neurotransmission is closely related to a wide variety of neurologic and psychiatric disorders, including anxiety, epilepsy, and schizophrenia 
(Macdonald et al., 2004; Charych et al., 2009). Recently, Li et al., 2016 reported that GABA-B receptors in the hippocampal dentate gyrus, which is involved in impairment of spatial learning and memory owing to vascular dementia, have been suggested as a potential new target for alternative treatment of cognitive dysfunction in vascular dementia. To date, the potential influence of GABA receptors on the treatment of focal cerebral I/R has not been studied. To investigate whether GABA-A or GABA-B receptors mediate neuroprotective effects of PNE, bicuculline (GABA-A receptor antagonist) and saclofen (GABA-B receptor antagonist) were used to inhibit GABA-A and GABA-B receptor activities, respectively. The results suggest that neuroprotective effects of B.L and PNE are significantly attenuated when coadministered with the GABA-B receptor antagonist saclofen, whereas the effect of GABA-A receptor antagonist bicuculline was not as obvious.

Thus, the findings of the present study suggest that I/R surgery alters intestinal flora and downregulates the population of B.L. The decrease in B.L levels then leads to downregulation of GABA receptor expression. After pretreatment with PNE, I/R-related shifts in intestinal flora can be prevented to some extent, and the relative abundance of B.L can be significantly enhanced. Enhanced B.L levels can then upregulate GABA-A and GABA-B receptor expression in the rat hippocampus, and the upregulated GABA-B receptors play a protective role in ischemic brain damage. To the best of our knowledge, this is the first report to elucidate cerebral protective effects of PNE that involve gut microbiota. It is important to note that B.L plays a key role in PNE treatment of cerebral I/R by upregulating GABA-B receptors.

\section{Acknowledgments}

The authors acknowledge Jiajia Shen, Yangfan Xu, Chong Chen, and Huimin Guo for contributions to study design and execution. Xiao Zheng and An Kang provided medical writing and editing support.

\section{Authorship Contributions}

Participated in research design: Liang, Wang, Xiao, H. Li, Liang, Wang.

Contributed new reagents or analytic tools: Shen, Zhu, Yin, Xie.

Conducted experiments: $\mathrm{H}$. Li, Xiao, X. Li, Chen, Kang.

Performed data analysis: Liang, H. Li, Xiao, X. LiY. Shao,

Wrote or contributed to the writing of the manuscript: Liang, $\mathrm{H}$. Li, Xiao, X. Li.

\section{References}

Barrett E, Ross RP, O'Toole PW, Fitzgerald GF, and Stanton C (2012) $\gamma$-Aminobutyric acid production by culturable bacteria from the human intestine. J Appl Microbiol 113: 411-417.

Bercik P, Park AJ, Sinclair D, Khoshdel A, Lu J, Huang X, Deng Y, Blennerhassett PA, Fahnestock M, Moine D, et al. (2011) The anxiolytic effect of Bifidobacterium longum NCC3001 involves vagal pathways for gut-brain communication. Neurogastroentero Motil 23:1132-1139.

Bharwani A, Mian MF, Surette MG, Bienenstock J, and Forsythe P (2017) Oral treatment with Lactobacillus rhamnosus attenuates behavioural deficits and immune changes in chronic social stress. BMC Med 15:7.

Björkholm C and Monteggia LM (2016) BDNF - a key transducer of antidepressant effects. Neuropharmacology 102:72-79.

Borrelli L, Aceto S, Agnisola C, De Paolo S, Dipineto L, Stilling RM, Dinan TG, Cryan JF, Menna LF, and Fioretti A (2016) Probiotic modulation of the microbiota-gut-brain axis and behaviour in zebrafish. Sci Rep 6:30046.

Braniste V, Al-Asmakh M, Kowal C, Anuar F, Abbaspour A, Tóth M, Korecka A, Bakocevic N, $\mathrm{Ng} \mathrm{LG}$, Kundu P, et al. (2014) The gut microbiota influences blood-brain barrier permeability in mice. Sci Transl Med 6:263ra158.

Bravo JA, Forsythe P, Chew MV, Escaravage E, Savignac HM, Dinan TG, Bienenstock J, and Cryan JF (2011) Ingestion of Lactobacillus strain regulates emotional behavior and centra GABA receptor expression in a mouse via the vagus nerve. Proc Natl Acad Sci USA 108: 16050-16055.

Charych EI, Liu F, Moss SJ, and Brandon NJ (2009) GABA(A) receptors and their associated proteins: implications in the etiology and treatment of schizophrenia and related disorders. Neuropharmacology 57:481-495.

Cheng CY, Su SY, Tang NY, Ho TY, Lo WY, and Hsieh CL (2010) Ferulic acid inhibits nitric oxide-induced apoptosis by enhancing GABA(B1) receptor expression in transient focal cerebral ischemia in rats. Acta Pharmacol Sin 31:889-899.

Clark A and Mach N (2016) Exercise-induced stress behavior, gut-microbiota-brain axis and diet: a systematic review for athletes. J Int Soc Sports Nutr 13:43.
Divyashri G, Krishna G, Muralidhara, and Prapulla SG (2015) Probiotic attributes, antioxidant, anti-inflammatory and neuromodulatory effects of Enterococcus faecium CFR 3003: in vitro and in vivo evidence. $J$ Med Microbiol 64:1527-1540.

Donnan GA, Fisher M, Macleod M, and Davis SM (2008) Stroke. Lancet 371:1612-1623.

Doyle KP, Simon RP, and Stenzel-Poore MP (2008) Mechanisms of ischemic brain damage. Neuropharmacology 55:310-318

Eisenstein M (2016) Microbiome: bacterial broadband. Nature 533:S104-S106.

El Aidy S, Dinan TG, and Cryan JF (2015) Gut microbiota: the conductor in the orchestra of immune-neuroendocrine communication. Clin Ther 37:954-967.

Fiorentino H, Kuczewski N, Diabira D, Ferrand N, Pangalos MN, Porcher C, and Gaiarsa JL (2009) $\mathrm{GABA}(\mathrm{B})$ receptor activation triggers BDNF release and promotes the maturation of GABAergic synapses. J Neurosci 29:11650-11661.

Foster JA, Lyte M, Meyer E, and Cryan JF (2016) Gut microbiota and brain function: an evolving field in neuroscience. Int J Neuropsychopharmacol 19:pyv114.

Fu CY, He XY, Li XF, Zhang X, Huang ZW, Li J, Chen M, and Duan CZ (2015) Nefiracetam attenuates pro-inflammatory cytokines and GABA transporter in specific brain regions of rats with post-ischemic seizures. Cell Physiol Biochem 37:2023-2031.

Gerriets T, Li F, Silva MD, Meng X, Brevard M, Sotak CH, and Fisher M (2003) The macrosphere model: evaluation of a new stroke model for permanent middle cerebral artery occlusion in rats. $J$ Neurosci Methods 122:201-211.

Guidance for Industry, Bioanalytical Method Validation (2001) US Department of Health and Human Services, Food and Drug Administration. Rockville, MD

Guo Q, Li P, Wang Z, Cheng Y, Wu H, Yang B, Du S, and Lu Y (2014) Brain distribution pharmacokinetics and integrated pharmacokinetics of Panax Notoginsenoside R1, Ginsenosides $\mathrm{Rg} 1, \mathrm{Rb} 1, \mathrm{Re}$ and $\mathrm{Rd}$ in rats after intranasal administration of Panax Notoginseng Saponins assessed by UPLC/MS/MS. J Chromatogr B Analyt Technol Biomed Life Sci 969:264-271.

Haiser HJ and Turnbaugh PJ (2012) Is it time for a metagenomic basis of therapeutics? Science 336:1253-1255.

Han D, Zhang QG, Yong-Liu, Li C, Zong YY, Yu CZ, Wang W, Yan JZ, and Zhang GY (2008) Coactivation of GABA receptors inhibits the JNK3 apoptotic pathway via the disassembly of the GluR6PSD95-MLK3 signaling module in cerebral ischemic-reperfusion. FEBS Lett 582:1298-1306.

Hao F, He Y, Sun Y, Zheng B, Liu Y, Wang X, Zhang Y, Lee RJ, Teng L, and Xie J (2016) Improvement of oral availability of ginseng fruit saponins by a proliposome delivery system containing sodium deoxycholate. Saudi J Biol Sci 23:S113-S125.

Helm KA, Haberman RP, Dean SL, Hoyt EC, Melcher T, Lund PK, and Gallagher M (2005) GABAB receptor antagonist SGS742 improves spatial memory and reduces protein binding to the cAMP response element (CRE) in the hippocampus. Neuropharmacology 48:956-964.

Huang XP, Ding H, Lu JD, Tang YH, Deng BX, and Deng CQ (2015) Effects of the combination of the main active components of Astragalus and Panax notoginseng on inflammation and apoptosis of nerve cell after cerebral ischemia-reperfusion. Am J Chin Med 43:1419-1438

Jeong JJ, Kim KA, Hwang YJ, Han MJ, and Kim DH (2016) Anti-inflammaging effects of Lactobacillus brevis OW38 in aged mice. Benef Microbes 7:707-718.

Joshi CN, Jain SK, and Murthy PS (2004) An optimized triphenyltetrazolium chloride method for identification of cerebral infarcts. Brain Res Brain Res Protoc 13:11-17.

Kim KA, Yoo HH, Gu W, Yu DH, Jin MJ, Choi HL, Yuan K, Guerin-Deremaux L, and Kim DH (2015) A prebiotic fiber increases the formation and subsequent absorption of compound $\mathrm{K}$ following oral administration of ginseng in rats. J Ginseng Res 39:183-187.

Ko CY, Lin HTV, and Tsai GJ (2013) Gamma-aminobutyric acid production in black soybean milk by Lactobacillus brevis FPA 3709 and the antidepressant effect of the fermented product on a forced swimming rat model. Process Biochem 48:559-568.

Lee WJ and Hase K (2014) Gut microbiota-generated metabolites in animal health and disease. Nat Chem Biol 10:416-424.

Li G, Lv J, Wang J, Wan P, Li Y, Jiang H, and Jin Q (2016) GABAB receptors in the hippocampal dentate gyrus are involved in spatial learning and memory impairment in a rat model of vascular dementia. Brain Res Bull 124:190-197.

Liu H, Yang J, Du F, Gao X, Ma X, Huang Y, Xu F, Niu W, Wang F, Mao Y, et al. (2009) Absorption and disposition of ginsenosides after oral administration of Panax notoginseng extract to rats. Drug Metab Dispos 37:2290-2298.

Longa EZ, Weinstein PR, Carlson S, and Cummins R (1989) Reversible middle cerebral artery occlusion without craniectomy in rats. Stroke 20:84-91.

Ma X, Peng JH, and Hu YY (2014) Chinese herbal medicine-induced liver injury. J clin Transl Hepatol 2:170-175.

Macdonald RL, Gallagher MJ, Feng HJ, and Kang J (2004) GABA(A) receptor epilepsy mutations. Biochem Pharmacol 68:1497-1506.

Maqsood R and Stone TW (2016) The gut-brain axis, BDNF, NMDA and CNS disorders. Neurochem Res $\mathbf{4 1 : 2 8 1 9 - 2 8 3 5 .}$

Mayer EA, Tillisch K, and Gupta A (2015) Gut/brain axis and the microbiota. J Clin Invest 125 926-938.

McGinnity CJ, Riaño Barros DA, Rosso L, Veronese M, Rizzo G, Bertoldo A, Hinz R, Turkheimer FE, Koepp MJ, and Hammers A (2017) Test-retest reproducibility of quantitative binding measures of $[(11) \mathrm{C}]$ Ro15-4513, a PET ligand for GABAA receptors containing alpha5 subunits. Neuroimage 152:270-282.

Meng J, Liu B, Li X, Yang Y, Liu S, Kong W, and Hu G (2012) Clinical effectiveness and safety of sanchi tong shu capsule in the treatment of aural vertigo: a multi-center randomized controlled clinical trial. Lin Chuang Er Bi Yan Hou Tou Jing Wai Ke Za Zhi 26:295-299.

Messaoudi M, Lalonde R, Violle N, Javelot H, Desor D, Nejdi A, Bisson JF, Rougeot C, Pichelin M, Cazaubiel M, et al. (2011a) Assessment of psychotropic-like properties of a probiotic formulation (Lactobacillus helveticus R0052 and Bifidobacterium longum R0175) in rats and human subjects. Br J Nutr 105:755-764.

Messaoudi M, Violle N, Bisson JF, Desor D, Javelot H, and Rougeot C (2011b) Beneficial psychological effects of a probiotic formulation (Lactobacillus helveticus R0052 and Bifidobacterium longum R0175) in healthy human volunteers. Gut Microbes 2:256-261.

Nasrallah FA, Singh KKDR, Yeow LY, and Chuang KH (2017) GABAergic effect on resting-state functional connectivity: dynamics under pharmacological antagonism. Neuroimage 149:53-62. Ohland CL, Kish L, Bell H, Thiesen A, Hotte N, Pankiv E, and Madsen KL (2013) Effects of Lactobacillus helveticus on murine behavior are dependent on diet and genotype and correlate with alterations in the gut microbiome. Psychoneuroendocrinology 38:1738-1747.

Ohsawa K, Uchida N, Ohki K, Nakamura Y, and Yokogoshi H (2015) Lactobacillus helveticusfermented milk improves learning and memory in mice. Nutr Neurosci 18:232-240. 
Sampson TR, Debelius JW, Thron T, Janssen S, Shastri GG, Ilhan ZE, Challis C, Schretter CE, Rocha S, Gradinaru V, et al. (2016) Gut microbiota regulate motor deficits and neuroinflammation in a model of parkinson's disease. Cell 167:1469-1480.e12.

Sampson TR and Mazmanian SK (2015) Control of brain development, function, and behavior by the microbiome. Cell Host Microbe 17:565-576.

Savignac HM, Kiely B, Dinan TG, and Cryan JF (2014) Bifidobacteria exert strain-specific effects on stress-related behavior and physiology in BALB/c mice. Neurogastroenterol Motil 26 $1615-1627$.

Shahidi S, Komaki A, Mahmoodi M, and Lashgari R (2008) The role of GABAergic transmission in the dentate gyrus on acquisition, consolidation and retrieval of an inhibitory avoidance learning and memory task in the rat. Brain Res 1204:87-93.

Teschke R, Wolff A, Frenzel C, Eickhoff A, and Schulze J (2015) Herbal traditional Chinese medicine and its evidence base in gastrointestinal disorders. World $J$ Gastroenterol 21: 4466-4490.

Tillisch K, Labus J, Kilpatrick L, Jiang Z, Stains J, Ebrat B, Guyonnet D, Legrain-Raspaud S, Trotin B, Naliboff B, et al. (2013) Consumption of fermented milk product with probiotic modulates brain activity. Gastroenterology 144:1394-1401, 1401.e1-4.

Vollenweider F, Bendfeldt K, Maetzler W, Otten U, and Nitsch C (2006) GABA(B) receptor expression and cellular localization in gerbil hippocampus after transient global ischemia. Neurosci Lett 395:118-123.

Vyas U and Ranganathan N (2012) Probiotics, prebiotics, and synbiotics: gut and beyond. Gastroenterol Res Pract 2012:872716.

Wallace BD, Wang H, Lane KT, Scott JE, Orans J, Koo JS, Venkatesh M, Jobin C, Yeh LA, Man $\mathrm{S}$, et al. (2010) Alleviating cancer drug toxicity by inhibiting a bacterial enzyme. Science $\mathbf{3 3 0}$ 831-835.

Wang GH, Jiang ZL, Fan XJ, Zhang L, Li X, and Ke KF (2007) Neuroprotective effect of taurine against focal cerebral ischemia in rats possibly mediated by activation of both GABAA and glycine receptors. Neuropharmacology 52:1199-1209.
Wang H, Lee IS, Braun C, and Enck P (2016) Effect of probiotics on central nervous system functions in animals and humans: a systematic review. J Neurogastroenterol Motil $\mathbf{2 2}$ $589-605$

Xiao J, Chen H, Kang D, Shao Y, Shen B, Li X, Yin X, Zhu Z, Li H, Rao T, et al. (2016) Qualitatively and quantitatively investigating the regulation of intestinal microbiota on the metabolism of panax notoginseng saponins. J Ethnopharmacol 194:324-336.

Xu R, Peng Y, Wang M, Fan L, and Li X (2014) Effects of broad-spectrum antibiotics on the metabolism and pharmacokinetics of ginsenoside Rb1: a study on rats gut microflora influenced by lincomycin. J Ethnopharmacol 158 Pt A:338-344.

Yang X, Xie L, Li Y, and Wei C (2009) More than 9,000,000 unique genes in human gut bacterial community: estimating gene numbers inside a human body. PLoS One 4:e6074.

Zheng G, Zhang X, Chen Y, Zhang Y, Luo W, and Chen J (2007) Evidence for a role of GABAA receptor in the acute restraint stress-induced enhancement of spatial memory. Brain Res 1181:61-73.

Zhou L, Xing R, Xie L, Rao T, Wang Q, Ye W, Fu H, Xiao J, Shao Y, Kang D, et al (2015) Development and validation of an UFLC-MS/MS assay for the absolute quantitation of nine notoginsenosides in rat plasma: application to the pharmacokinetic study of Panax Notoginseng Extract. J Chromatogr B Analyt Technol Biomed Life Sci 995 996:46-53

Address correspondence to: Dr. Guangji Wang, Key Laboratory of Drug Metabolism and Pharmacokinetics, State Key Laboratory of Natural Medicines, Tongjiaxiang 24, Nanjing 210009, China; E-mail: guangjiwang@hotmail.com. Or, Dr. Yan Liang, China Pharmaceutical University, Tongjiaxiang 24, Nanjing 210009, China. E-mail: liangyan0679@163.com 